\title{
Spectrally Precoded OFDM
}

\author{
Char-Dir Chung, Senior Member, IEEE
}

\begin{abstract}
By bilinearly transforming the rectangularly pulsed multicarrier basis signals, new basis sets containing full-response continuous basis signals are developed for constructing spectrally efficient orthogonal frequency-division multiplexing (OFDM) signals with or without cyclic prefix (CP) or zero padding (ZP). Both multiple-product and linear-combination formats are presented for these basis sets. In the multiple-product format, the developed full-response basis signals are shown to be zero-valued at interval edges, and thus can be used to construct continuous-phase OFDM signals. It is analytically shown that the constructed continuous-phase OFDM signals exhibit relatively small power spectral sidelobes which fall off as $f^{-4}$, and can thus provide much higher spectral efficiency than the traditional rectangularly pulsed OFDM signal. In the linear-combination format, the proposed basis signals are shown to be the linear combinations of rectangularly pulsed multicarrier basis signals, and thereby allow for the efficient realization of fast Fourier transform (FFT). Specifically, a spectrally precoded signaling structure is developed in conjunction with inverse FFT and guard insertion to construct continuous-phase OFDM signals with or without $C P$ or $Z P$. The corresponding spectral decoders are also developed to enable zero-forcing (ZF) demodulation for spectrally precoded OFDM. With the zero-forcing demodulation, the spectrally precoded OFDM schemes are shown to outperform in average error-rate characteristics the conventional rectangularly pulsed and correlatively coded OFDM schemes on various delay-spread channels.
\end{abstract}

Index Terms-Cyclic prefix (CP), fast Fourier transform (FFT), orthogonal frequency-division multiplexing (OFDM), spectral precoding, zero-padding (ZP).

\section{INTRODUCTION}

$\mathbf{O}$ RTHOGONAL frequency-division multiplexing (OFDM) [1]-[23] has been well known to guarantee reliable communications over channels with impulsive noise, dispersion, and/or multipath fading, and adopted as the modulation method in many contemporary broadband communication applications [27]-[29]. Most applications entail redundant block transmission and enable efficacious block processes to counteract the adverse channel effects by inserting guard intervals with cyclic prefix (CP) [4]-[8] or zero-padding (ZP) [9] among useful data blocks. Since block processes can be efficiently implemented using fast Fourier transform (FFT) algorithms [16], a large number of multiplexed subcarriers (sometimes, hundreds) is

Paper approved by J. Wang, the Editor for Wireless Spread Spectrum of the IEEE Communications Society. Manuscript received April 22, 2005; revised December 30, 2005, March 16, 2006, and April 6, 2006. This work was supported in part by the R.O.C. National Science Council under Council 94-2213-E-002-128.

The author is with the Department of Electrical Engineering and the Graduate Institute of Communications Engineering, National Taiwan University, Taipei, Taiwan 10617, R.O.C. (e-mail: cdchung@cc.ee.ntu.edu.tw)

Digital Object Identifier 10.1109/TCOMM.2006.884850 commonly used for embodying OFDM so that the channel counteraction is exploited to a large extent.

Pulse-shaping is important in the OFDM system design. Most practical approaches use rectangular pulses [4]-[15] because such a pulse shape is very suitable for use in conjunction with the FFT process and guard insertion. Unfortunately, the rectangularly pulsed OFDM possesses discontinuous pulse edges and exhibits relatively large power spectral sidelobes which fall off as $f^{-2}$ [15]. To minimize signal distortion over bandlimited channels and power spillover into adjacent channels, the subcarriers at the band edges are not modulated for most applications [27]-[29], thus reducing the achievable spectral efficiency. Although smoother pulses of finite duration [17]-[19] or infinite duration [1]-[3], [21], [22] have been suggested for OFDM, these different pulses are not widely adopted in practice, because implementation of FFT becomes either impossible or very complex (e.g., [3]), and guard insertion is sometimes infeasible when the pulses other than the rectangular one are used. In order to increase spectral compactness, lowpass filtering after the inverse FFT (IFFT) process and guard insertion is pragmatically applied to the rectangularly pulsed OFDM signal in practical transmitters [20]. This, however, breaks the orthogonality among multiplexed subcarriers, and sometimes, consecutive blocks, and entails noticeable degradation in error performance, especially on dispersion and multipath fading channels.

Precoded OFDM is a popular signaling structure adopted in many studies to enhance various performance characteristics of rectangularly pulsed OFDM while simultaneously enabling the FFT process and guard insertion [6]-[8], [10]-[15]. By delicately introducing correlation within data blocks in the frequency domain, many precoded OFDM schemes have been devised to counteract channel effects [6]-[8], suppress intercarrier interference [10], [11], reduce peak-to-average-power ratio [12]-[14], and increase spectral compactness [15]. Particularly, a class of correlatively $\mathcal{G}_{L}$-coded OFDM schemes is first proposed in [15] to significantly improve the spectral compactness of rectangularly pulsed OFDM. These schemes are applicable to the OFDM signals without guard intervals (NG-OFDM) with $\mathrm{CP}(\mathrm{CP}-\mathrm{OFDM})$, and with ZP (ZP-OFDM). $\mathcal{G}_{L}$-coded NG-OFDM and ZP-OFDM signals are analytically shown to possess extremely small power spectral sidelobes which roll off very fast as $f^{-2 L-2}$. As a result of this property, much higher spectral efficiency than rectangularly pulsed OFDM is achieved by $\mathcal{G}_{L}$-coded OFDM, and the improvement increases with $L$. However, because correlative coding breaks the orthogonality among multiplexed data-modulated waveforms, $\mathcal{G}_{L}$-coded OFDM degrades remarkably in error performance from rectangularly pulsed OFDM on the additive white Gaussian noise (AWGN) channel and various delay-spread (DS) channels with continuous spreads, and the degradation increases with $L$. 
In the paper, a new class of spectrally precoded schemes is proposed to improve the spectral compactness of rectangularly pulsed OFDM without trading off error performance. The key idea here is to develop new basis signal sets for OFDM, which can achieve high spectral compactness while enabling realization in the precoded OFDM structure. Because the proposed schemes are constructed from new basis sets, the orthogonality among multiplexed data-modulated waveforms on useful data intervals is sustained, thus warranting the error-performance prevalence achieved by OFDM. The new basis sets are developed in Section II by bilinearly transforming the conventional multicarrier basis set that constructs the rectangularly pulsed OFDM signal. Because full-response continuous basis signals with zero interval edges are contained, these basis sets enable the construction of continuous-phase OFDM signals. The corresponding precoded OFDM structure is formulated in Section III. Several spectrally precoded NG-OFDM, CP-OFDM, and ZP-OFDM signals are shown to possess small power spectral sidelobes which roll off as $f^{-4}$. A zero-forcing $(\mathrm{ZF})$ receiving scheme is given in Section IV for demodulating the spectrally precoded OFDM signals, and compared with uncoded and $\mathcal{G}_{L}$-coded OFDM schemes in error performance. Section $\mathrm{V}$ concludes the paper.

\section{USEFUl BASIS SignAl SETS}

A continuous-phase modulation signal is commonly referred to in the literature [21]-[25] as the digitally modulated signal that is continuous on the time domain. Due to phase continuity, continuous-phase signals are known to exhibit fast spectral rolloff at band edges in both single-carrier [23], [25] and multicarrier [21]-[24] modulations. Particularly in [21] and [22], the NG-OFDM signal is generally formulated in terms of the orthogonal multiplexing of linearly data-modulated basis signals, and shown to have the power spectral density (PSD) in the general form of a sum of the squared magnitudes of the Fourier transforms of basis signals. The general signaling format indicates that the continuous-phase NG-OFDM signal can be constructed by using the full-response basis signals that are continuous on $|t| \leq T_{d} / 2$ and zero-valued at both edges $t= \pm T_{d} / 2$, with $T_{d}$ denoting the time length for sending a useful OFDM data block. ${ }^{1}$ As a result, the PSD of the continuous-phase NG-OFDM signal is determined by such constituent basis signals, and thus exhibits small power spectral sidelobes which roll off as $f^{-4}$ or faster [26]. Because the $\mathrm{ZP}-\mathrm{OFDM}$ signal is zero-valued in guard intervals, the basis signal sets constructing continuous-phase NG-OFDM signals can also build up the continuous-phase ZP-OFDM signals exhibiting small power spectral sidelobes. Such basis signal sets are exploited below.

Denote $\Psi$ as the multicarrier basis set that expands the rectangularly pulsed OFDM signal using $N$ radian frequencies equally spaced by $\omega_{d} \triangleq 2 \pi / T_{d}$. When $N$ is a positive integer power of

\footnotetext{
${ }^{1}$ In the paper, a signal set is called a basis set if all the element signals are orthonormal in the sense that they have unit energy and are mutually orthogonal on $|t| \leq T_{d} / 2$. Two disjoint signal sets are said to be orthonormal if all the element signals are orthonormal on $|t| \leq T_{d} / 2$.
}

two and $\omega_{0}$ is an integer multiple of $\omega_{d}$ with $\omega_{0} \gg \omega_{d}$, $\Psi$ can be expressed as ${ }^{2}$

$$
\Psi \triangleq\left\{C_{n}^{(0)}(t), S_{n}^{(0)}(t) ; n \in \mathcal{Z}_{N}\right\}
$$

on $|t| \leq T_{d} / 2$, where $C_{n}^{(m)}(t)$ and $S_{n}^{(m)}(t)$ are the quadrature carriers with frequency $\omega_{0}+(1 / 2) m \omega_{d}+n \omega_{d}$, defined by $C_{n}^{(m)}(t) \triangleq \sqrt{2 / T_{d}} \cos \left(\left(\omega_{0}+(1 / 2) m \omega_{d}+n \omega_{d}\right) t\right)$ and $S_{n}^{(m)}(t) \triangleq \sqrt{2 / T_{d}} \sin \left(\left(\omega_{0}+(1 / 2) m \omega_{d}+n \omega_{d}\right) t\right)$, for $n \in$ $\mathcal{Z}_{N}$ and $m \in \mathcal{Z}_{2}$. Note that $\Psi$ is exactly the basis set $\Omega_{2}$ in [21]. Because one-half of the basis signals (i.e., $C_{n}^{(0)}(t) \mathrm{s}$ ) are not zero-valued at edges $t= \pm T_{d} / 2$, the NG-OFDM and ZP-OFDM signals based on $\Psi$ are discontinuous in phase and exhibit relatively large power spectral sidelobes which fall off as $f^{-2}$. Nevertheless, the other half of the basis signals in $\Psi$ (i.e., $S_{n}^{(0)}(t)$ 's) are both continuous on $|t| \leq T_{d} / 2$ and zero-valued at edges $t= \pm T_{d} / 2$. In what follows, we intend to construct from $\Psi$ some new basis sets containing more basis signals which are continuous on $|t| \leq T_{d} / 2$ and also zero-valued at edges $t= \pm T_{d} / 2$. These new basis sets are obtained by applying a bilinear transform to $\Psi$ and its transform output sequentially. Specifically, the bilinear transform is defined by converting a $2 K$-dimensional ( $2 K$-D) basis set $\left\{h_{k}(t) ; k \in \mathcal{Z}_{2 K}\right\}$ into another $2 K$-D set by

$$
\left\{h_{k}(t) ; k \in \mathcal{Z}_{2 K}\right\} \Rightarrow\left\{\frac{1}{\sqrt{2}}\left(h_{2 k}(t) \pm h_{2 k+1}(t)\right) ; k \in \mathcal{Z}_{K}\right\}_{(2)}
$$

Because the orthonormality is preserved with the transform, the output set is also a $2 K$-D basis set. Moreover, if the basis signals in the input set are continuous on $|t| \leq T_{d} / 2$, so are the basis signals in the output set. Nevertheless, the bilinear transform may generate a new basis signal that takes zero value at edges $t= \pm T_{d} / 2$, even though its constituent original basis signals are not zero-valued at the edges. This is the key idea behind the use of the bilinear transform for developing more zero-edged continuous basis signals. Conveniently enough, we classify the new basis sets into two families, as follows.

\section{A. Family $\mathcal{W}$}

Define the following signal sets that contain continuous signals on $|t| \leq T_{d} / 2$ :

$$
\begin{aligned}
\Phi_{1}^{(c) \triangleq} & \left\{P_{1}^{(c)}(t) C_{2 n}^{(1)}(t), P_{1}^{(c)}(t) S_{2 n}^{(1)}(t) ; n \in \mathcal{Z}_{N / 2}\right\} \\
\Phi_{u}^{(c) \triangleq} & \left\{P_{1}^{(s)}(t)\left(\prod_{k=1}^{u-2} P_{2^{k}}^{(c)}(t)\right) P_{2^{u-1}}^{(s)}(t) C_{n 2^{u}+\zeta(u)}^{(1)}(t),\right. \\
& P_{1}^{(s)}(t)\left(\prod_{k=1}^{u-2} P_{2^{k}}^{(c)}(t)\right) P_{2^{u-1}}^{(s)}(t) S_{n 2^{u}+\zeta(u)}^{(1)}(t) ; \\
& \left.n \in \mathcal{Z}_{N / 2^{u}}\right\}, \quad u=2,3, \ldots, \log _{2} N
\end{aligned}
$$

${ }^{2}$ Throughout the paper, we denote $\mathcal{Z}_{K} \triangleq\{0,1, \ldots, K-1\}$ 


$$
\begin{gathered}
\Phi_{u}^{(d) \triangleq}\left\{P_{1}^{(s)}(t)\left(\prod_{k=1}^{u-1} P_{2^{k}}^{(c)}(t)\right) C_{n 2^{u}+\zeta(u)}^{(1)}(t)\right. \\
\left.P_{1}^{(s)}(t)\left(\prod_{k=1}^{u-1} P_{2^{k}}^{(c)}(t)\right) S_{n 2^{u}+\zeta(u)}^{(1)}(t) ; n \in \mathcal{Z}_{N / 2^{u}}\right\} \\
u=1,2, \ldots, \log _{2} N
\end{gathered}
$$

where $P_{n}^{(c)}(t) \triangleq \sqrt{2} \cos \left((1 / 2) n \omega_{d} t\right), \quad P_{n}^{(s)}(t) \triangleq$ $\sqrt{2} \sin \left((1 / 2) n \omega_{d} t\right)$, and $\zeta(u) \triangleq \sum_{k=0}^{u-2} 2^{k}=2^{u-1}-1 .^{3}$ By applying the bilinear transform (2) to $\Psi$ and its transform output sequentially, various unions of these sets are shown to form basis sets as follows.

Lemma 1: For $u \in\left\{1,2, \ldots, \log _{2} N\right\}, \Phi_{u}^{(c)}$ and $\Phi_{u}^{(d)}$ are two orthonormal $2^{1-u} N$-D basis sets and form a $2^{2-u} N$-D basis set.

Proof: For $u=1, \Phi_{1}^{(c)} \cup \Phi_{1}^{(d)}$ is the output set after applying (2) to $\Psi$ with $h_{n}(t)=C_{n}^{(0)}(t)$ and $h_{N+n}(t)=S_{n}^{(0)}(t)$ for $n \in \mathcal{Z}_{N}$, through the aid of (32)-(35) with $\alpha=0.4$ Since $\Psi$ is a $2 N$-D basis set, the transform output $\Phi_{1}^{(c)} \cup \Phi_{1}^{(d)}$ constitutes a $2 N$-D basis set. Thus, $\Phi_{1}^{(c)}$ and $\Phi_{1}^{(d)}$ are orthonormal $N$-D basis sets. For $u \in\left\{2,3, \ldots, \log _{2} N\right\}$, $\Phi_{u}^{(c)} \cup \Phi_{u}^{(d)}$ can be obtained by applying (2) to $\Phi_{u-1}^{(d)}$ with $h_{n}(t)=P_{1}^{(s)}(t)\left(\prod_{k=1}^{u-2} P_{2^{k}}^{(c)}(t)\right) C_{n 2^{u-1}+\zeta(u-1)}^{(1)}(t)$ and $h_{N / 2^{u-1}+n}(t)=P_{1}^{(s)}(t)\left(\prod_{k=1}^{u-2} P_{2^{k}}^{(c)}(t)\right) S_{n 2^{u-1}+\zeta(u-1)}^{(1)}(t)$ for $n \in \mathcal{Z}_{N / 2^{u-1}}$, through the aid of (32)-(35) with $\alpha=1$. Since $\Phi_{u-1}^{(d)}$ is a $2^{2-u} N$-D basis set, so is the transform output $\Phi_{u}^{(c)} \cup \Phi_{u}^{(d)}$. It follows that $\Phi_{u}^{(c)}$ and $\Phi_{u}^{(d)}$ are orthonormal $2^{1-u} N$-D basis sets.

Lemma 2: $\Phi_{1}^{(c)}, \Phi_{2}^{(c)}, \ldots, \Phi_{\log _{2} N}^{(c)}$ are mutually orthonormal.

Proof: It can be inferred from the proof of Lemma 1 that, for $u \in\left\{2,3, \ldots, \log _{2} N\right\}, \Phi_{u}^{(c)}$ is orthonormal to $\cup_{i=1}^{u-1} \Phi_{i}^{(c)}$ because $\Phi_{u}^{(c)}$ contains basis signals residing in the space spanned by $\Phi_{u-1}^{(d)}$, and $\cup_{i=1}^{u-1} \Phi_{i}^{(c)}$ is orthonormal to $\Phi_{u-1}^{(d)}$. The lemma is thus proven by induction.

Because $P_{1}^{(s)}\left( \pm T_{d} / 2\right) \neq 0$ and $P_{2^{k}}^{(c)}\left( \pm T_{d} / 2\right) \neq 0$ for positive integer $k$, all the signals in $\Phi_{u}^{(d)}$, s are not zero-valued at $t=$ $\pm T_{d} / 2$ and are thus obsolete. However, since $P_{1}^{(c)}\left( \pm T_{d} / 2\right)=0$ and $P_{2^{k}}^{(s)}\left( \pm T_{d} / 2\right)=0$ for positive integer $k$, all the continuous signals in $\Phi_{u}^{(c)}$,s are zero-valued at $t= \pm T_{d} / 2$ and are thus useful. In conjunction with Lemma 2, this indicates that any union of $\Phi_{1}^{(c)}, \Phi_{2}^{(c)}, \ldots, \Phi_{\log _{2} N}^{(c)}$ forms a basis set containing zero-edged continuous basis signals on $|t| \leq T_{d} / 2$. Accordingly, the family of basis sets $\left\{\mathcal{W}_{L} ; L=1,2, \ldots, \log _{2} N\right\}$ is thus proposed.

Proposition 1: For $L \in\left\{1,2, \ldots, \log _{2} N\right\}, \mathcal{W}_{L} \triangleq$ $\cup_{u=1}^{L} \Phi_{u}^{(c)}$ is a $2 M$-D basis set containing zero-edged continuous basis signals on $|t| \leq T_{d} / 2$, where $M \triangleq \sum_{i=1}^{L} 2^{-i} N=$ $N\left(1-2^{-L}\right)$.

Notably, $\mathcal{W}_{1}$ is exactly the $N$-D $\Omega_{4}(1)$ in [21]. Except $\mathcal{W}_{1}$, the other $\mathcal{W}_{L}$ 's are new basis sets that were never exploited.

\footnotetext{
${ }^{3}$ By default, we set $\sum_{i=m}^{n} f_{i}=0$ and $\prod_{i=m}^{n} f_{i}=1$ if $m>n$.

${ }^{4}$ For notational brevity, sign inversion has been applied when deriving $\Phi_{1}^{(d)}$ and $\Phi_{u}^{(c)}$ with $u>1$ by quoting (35). This does not, however, affect the orthonormality characteristics of the signals contained in these sets.
}

\section{B. Family $\mathcal{V}$}

Consider the following sets that contain continuous signals on $|t| \leq T_{d} / 2$ :

$$
\begin{aligned}
& \Theta_{u}^{(c)} \triangleq\left\{P_{N / 2^{u}}^{(s)}(t) \prod_{k=1}^{u-1} P_{N / 2^{k}}^{(c)}(t) C_{n+\frac{1}{2} \chi(u+1)}^{(0)}(t),\right. \\
& P_{N / 2^{u}}^{(s)}(t) \prod_{k=1}^{u-1} P_{N / 2^{k}}^{(c)}(t) S_{n+\frac{1}{2} \chi(u+1)}^{(0)}(t) ; \\
& \left.n \in \mathcal{Z}_{N / 2^{u}}\right\}, \quad u=1,2, \ldots, \log _{2} N-1 \\
& \Theta_{\log _{2} N}^{(c)} \triangleq\left\{\prod_{k=1}^{\log _{2} N} P_{N / 2^{k}}^{(c)}(t) C_{n+\frac{1}{2} \chi\left(\log _{2} N\right)}^{(1)}(t),\right. \\
& \left.\prod_{k=1}^{\log _{2} N} P_{N / 2^{k}}^{(c)}(t) S_{n+\frac{1}{2} \chi\left(\log _{2} N\right)}^{(1)}(t)\right\} \\
& \Theta_{u}^{(d)} \triangleq\left\{\prod_{k=1}^{u} P_{N / 2^{k}}^{(c)}(t) C_{n+\frac{1}{2} \chi(u+1)}^{(0)}(t),\right. \\
& \left.\prod_{k=1}^{u} P_{N / 2^{k}}^{(c)}(t) S_{n+\frac{1}{2} \chi(u+1)}^{(0)}(t) ; n \in \mathcal{Z}_{N / 2^{u}}\right\} \\
& u=1,2, \ldots, \log _{2} N-1 \\
& \Theta_{\log _{2} N}^{(d)} \triangleq\left\{P_{1}^{(s)}(t) \prod_{k=1}^{\log _{2} N-1} P_{N / 2^{k}}^{(c)}(t) C_{\frac{1}{2} \chi\left(\log _{2} N\right)}^{(1)}(t),\right. \\
& \left.P_{1}^{(s)}(t) \prod_{k=1}^{\log _{2} N-1} P_{N / 2^{k}}^{(c)}(t) S_{\frac{1}{2} \chi\left(\log _{2} N\right)}^{(1)}(t)\right\}
\end{aligned}
$$

with $\chi(u) \triangleq \sum_{k=1}^{u-1} N / 2^{k}=N\left(1-2^{1-u}\right)$. Analogous to the derivation of $\Phi_{u}^{(c)}$,s and $\Phi_{u}^{(d)}$, s, $\Theta_{u}^{(c)} \cup \Theta_{u}^{(d)}$ can be obtained as the output set after applying the bilinear transform (2) with the aid of (32)-(35). For example, when $u \in\left\{2,3, \ldots, \log _{2} N-1\right\}$, $\Theta_{u}^{(c)} \cup \Theta_{u}^{(d)}$ is the result after bilinearly transforming $\Theta_{u-1}^{(d)}$ with $h_{2 n}(t)=\prod_{k=1}^{u-1} P_{N / 2^{k}}^{(c)}(t) C_{n+\chi(u) / 2}^{(0)}(t), h_{2 n+1}(t)=$ $\prod_{k=1}^{u-1} P_{N / 2^{k}}^{(c)}(t) C_{n+\chi(u) / 2+N / 2^{u}}^{(0)}(t), h_{N / 2^{u-1}+2 n}(t)=\prod_{k=1}^{u-1}$ $P_{N / 2^{k}}^{(c)}(t) S_{n+\chi(u) / 2}^{(0)}(t)$, and $h_{N / 2^{u-1}+2 n+1}(t)=\prod_{k=1}^{u-1} P_{N / 2^{k}}^{(c)}$ $(t) S_{n+\chi(u) / 2+N / 2^{u}}^{(0)}(t)$ for $n \in \mathcal{Z}_{N / 2^{u}}$. Therefore, the following two lemmas can be likewise proved as Lemmas 1 and 2.

Lemma 3: For $u \in\left\{1,2, \ldots, \log _{2} N\right\}, \Theta_{u}^{(c)}$ and $\Theta_{u}^{(d)}$ are two orthonormal $2^{1-u} N$-D basis sets and form a $2^{2-u} N$-D basis set. Lemma 4: $\Theta_{1}^{(c)}, \Theta_{2}^{(c)}, \ldots, \Theta_{\log _{2} N}^{(c)}$ are mutually orthonormal. Because $P_{1}^{(c)}\left( \pm T_{d} / 2\right)=0, P_{1}^{(s)}\left( \pm T_{d} / 2\right) \neq 0$, $P_{2^{k}}^{(s)}\left( \pm T_{d} / 2\right)=0$, and $P_{2^{k}}^{(c)}\left( \pm T_{d} / 2\right) \neq 0$ for positive integer $k$, all the continuous signals in $\Theta_{u}^{(c)}$ 's are zero-valued at $t= \pm T_{d} / 2$ and useful, but those in $\Theta_{u}^{(d)}$, s are not. From Lemma 4 , it follows that any union of $\Theta_{1}^{(c)}, \Theta_{2}^{(c)}, \ldots$, and $\Theta_{\log _{2} N}^{(c)}$ forms a basis set containing zero-edged continuous basis signals on $|t| \leq T_{d} / 2$. We thus propose the new family $\left\{\mathcal{V}_{L} ; L=1,2, \ldots, \log _{2} N\right\}$. 
Proposition 2: For $L \in\left\{1,2, \ldots, \log _{2} N\right\}, \mathcal{V}_{L} \triangleq \cup_{u=1}^{L} \Theta_{u}^{(c)}$ is a $2 M$-D basis set containing zero-edged continuous basis signals on $|t| \leq T_{d} / 2$.

Consider the basis signals in $\mathcal{V}_{L}$ on the extended interval $-\left(T_{d} / 2\right)-\left(T_{d} / 2^{\varpi}\right) \leq t \leq\left(T_{d} / 2\right)$ for $L<\log _{2} N$ and positive integer $\varpi$. Obviously, these signals are still continuous on the extended interval. Because $P_{2^{u}}^{(s)}\left(-\left(T_{d} / 2\right)-\left(T_{d} / 2^{\varpi}\right)\right)=0$ for positive integers $u$ and $\varpi$ satisfying $\varpi \leq u$, these signals appear to be zero-valued at $t=-\left(T_{d} / 2\right)-\left(T_{d} / 2^{\varpi}\right)$ as well, if $L<\log _{2} N$ and $\varpi \in\left\{1,2, \ldots, \log _{2} N-L\right\}$. This enables the construction of continuous-phase CP-OFDM signals.

\section{Alternative Formats for $\mathcal{W}_{L}$ and $\mathcal{V}_{L}$}

The above expressions in (3), (4), (6), and (7) can be referred to as the multiple-product formats for $\mathcal{W}_{L}$ and $\mathcal{V}_{L}$, in that the basis signals are formulated in terms of the product of multiple pulses and carrier signals. Such formats are useful in the sense that all the continuous basis signals are explicitly shown to be zero-valued at interval edges, and thus suited to compose continuous-phase NG-OFDM and ZP-OFDM signals. However, the multiple-product formats are themselves too complicated to realize the real waveform and to analyze the spectral characteristics. To overcome the inconvenience, alternative expressions for $\Phi_{u}^{(c)}$ 's and $\Theta_{u}^{(c)}$, s are derived in Appendices B and C as

$$
\begin{aligned}
& \Phi_{u}^{(c)}=\left\{2^{-\frac{u}{2}} \sum_{v=0}^{2^{u}-1}(-1)^{1+\psi_{u, v}} C_{n 2^{u}+v}^{(0)}(t),\right. \\
&\left.2^{-\frac{u}{2}} \sum_{v=0}^{2^{u}-1}(-1)^{1+\psi_{u, v}} S_{n 2^{u}+v}^{(0)}(t) ; n \in \mathcal{Z}_{\frac{N}{2^{u}}}\right\} \\
& \Theta_{u}^{(c)}=\left\{2^{-\frac{u}{2}} \sum_{v=0}^{2^{u}-1} \phi_{u, v} C_{n+\frac{N}{2^{u} v}}^{(0)}(t),\right. \\
&\left.2^{-\frac{u}{2}} \sum_{v=0}^{2^{u}-1} \phi_{u, v} S_{n+\frac{N}{2^{u}} v}^{(0)}(t) ; n \in \mathcal{Z}_{\frac{N}{2^{u}}}\right\}
\end{aligned}
$$

on $|t| \leq T_{d} / 2$ for $u=1,2, \ldots, \log _{2} N$. In (10), $\psi_{u, v}$ is the sum of the most and least significant bits in the binary representation (in $u$ bits) of the modulo- $2^{u}$ value of $v$ when $u \geq 2$ and $\psi_{1, v}=$ 1 by default. In (11), $\phi_{u, v}=1$ if $u=\log _{2} N$ and $\phi_{u, v}=$ $(-1)^{\varsigma_{v}}$ otherwise, where $\varsigma_{v}$ represents the least significant bit in the binary representation of $v$. These alternative expressions are expressed in terms of the linear combinations of carrier signals, and thus referred to as the linear-combination formats for $\mathcal{W}_{L}$ and $\mathcal{V}_{L}$. These formats are used below to facilitate both practical realization and spectral analysis of the OFDM signals based on $\mathcal{W}_{L}$ and $\mathcal{V}_{L}$

\section{SPeCtrally PReCoded OFDM}

Consider the memoryless source that generates a block of $M$ complex data symbols simultaneously and independently every $T$ seconds, say $\left\{D_{m, k}\right\}_{m=0}^{M-1}$ for the $k$ th signaling interval $k T-T_{g}-T_{d} / 2 \leq t<k T+T_{d} / 2$, with $T=T_{d}+T_{g}$. Here, $T_{g}$ represents the length of the time interval for inserting
CP or ZP. All the data symbols $D_{m, k}$ 's are assumed to be independent and identically distributed (i.i.d.) with zero mean and $E\left\{\left|D_{m, k}\right|^{2}\right\}=1$. Decomposing $D_{m, k}=D_{m, k}^{(I)}-j D_{m, k}^{(Q)}$, the OFDM signals constructed from $\mathcal{W}_{L}$ and $\mathcal{V}_{L}$ in the linear-combination format can be represented by [21]

$$
\begin{aligned}
s(t)= & \rho \sqrt{\frac{T_{d}}{2}} \sum_{k} p(t-k T) \\
& \cdot\left\{\sum_{u=1}^{L} 2^{-\frac{u}{2}} \sum_{n=0}^{\frac{N}{2^{u}}-1} \sum_{v=0}^{2^{u}-1}(-1)^{1+\psi \psi_{u, v}}\right. \\
& \times\left[D_{\chi(u)+n, k}^{(I)} C_{n 2^{u}+v}^{(0)}(t-k T)\right. \\
& \left.\left.+D_{\chi(u)+n, k}^{(Q)} S_{n 2^{u}+v}^{(0)}(t-k T)\right]\right\}, \\
s(t)= & \rho \sqrt{\frac{T_{d}}{2} \sum_{k} p(t-k T)} \\
& \cdot\left\{\sum_{u=1}^{L} 2^{-\frac{u}{2}} \sum_{n=0}^{\frac{N}{2 u}-1} \sum_{v=0}^{2^{u}-1} \phi_{u, v}\right. \\
& \times\left[D_{\chi(u)+n, k}^{(I)} C_{n+\frac{N}{2^{u}} v}^{(0)}(t-k T)\right. \\
& \left.\left.+D_{\chi(u)+n, k}^{(Q)} S_{n+\frac{N}{2^{u} v}}^{(0)}(t-k T)\right]\right\}, \\
& \text { for } \mathcal{V}_{L}-\text { based OFDM. OFDM }
\end{aligned}
$$

Here, $\rho$ is the signaling amplitude. The windowing function $p(t)$ is defined on $-T_{g}-T_{d} / 2 \leq t<T_{d} / 2$. If $s(t)$ models the CP-OFDM signal, $p(t)$ is a unit rectangular pulse on $-T_{g}-$ $T_{d} / 2 \leq t<T_{d} / 2$ and basis signals are applied on each (extended) interval $k T-T_{g}-T_{d} / 2 \leq t<k T+T_{d} / 2$. If $s(t)$ models the ZP-OFDM signal, $p(t)$ is a unit rectangular pulse on $-T_{d} / 2 \leq t<T_{d} / 2$ and $p(t)=0$ elsewhere. In the latter case, basis signals are applied only on each useful signaling interval $k T-T_{d} / 2 \leq t<k T+T_{d} / 2$. By modeling so, the complex data symbol time $T_{s}$ is related to the OFDM block length $T$ by $T_{s}=T / M$.

When $T_{g}=0$, both (12) and (13) also model the NG-OFDM signals. Because $\mathcal{W}_{L}$ and $\mathcal{V}_{L}$ contain zero-edged continuous signals on $|t| \leq T_{d} / 2$, the ZP-OFDM and NG-OFDM signals in (12) and (13) are continuous for all $t$, and thus form continuous-phase signals. Moreover, if $\varpi \in\left\{1,2, \ldots, \log _{2} N-L\right\}$ and $L<\log _{2} N$, because all the signals in $\mathcal{V}_{L}$ are still continuous and zero-edged on the extended interval $-\left(T_{d} / 2\right)-$ $\left(T_{d} / 2^{\varpi}\right) \leq t \leq\left(T_{d} / 2\right)$, the $\mathcal{V}_{L}$-based CP-OFDM signal in (13) with $T_{g}=2^{-\varpi} T_{d}$ is continuous for all $t$ and also forms a continuous-phase signal.

The above signal models can be unified as

$$
\begin{array}{r}
s(t)=\rho \sum_{k} \Re\left\{\sum_{n=0}^{N-1} B_{n, k} \exp \left\{j\left(\omega_{0}+n \omega_{d}\right)(t-k T)\right\}\right\} \\
\times p(t-k T)
\end{array}
$$




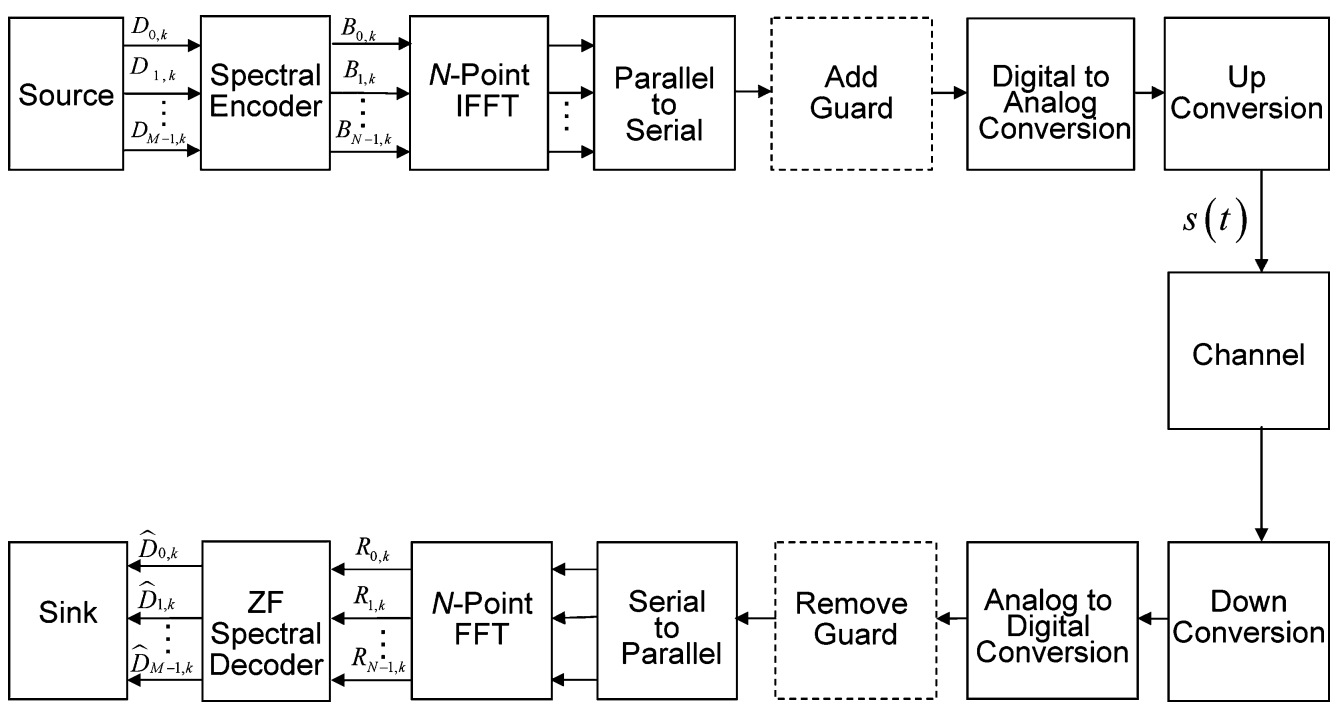

Fig. 1. System model.

where $\left\{B_{n, k}\right\}_{n=0}^{N-1}$ is related to $\left\{D_{m, k}\right\}_{m=0}^{M-1}$ by the mapping

$$
B_{n, k}=\sum_{m=0}^{M-1} G_{n, m}^{(\mathcal{X})} D_{m, k}, \quad n \in \mathcal{Z}_{N}
$$

for $\mathcal{X}=\mathcal{W}$ or $\mathcal{V}$. Here, $G_{n, m}^{(\mathcal{X})}$ 's denote the coefficients, given by

$$
G_{n 2^{u}+v, \chi(u)+n}^{(\mathcal{W})}=2^{-\frac{u}{2}}(-1)^{1+\psi_{u, v}}, \quad n \in \mathcal{Z}_{N / 2^{u}} \text { and } v \in \mathcal{Z}_{2^{u}}
$$

for $u=1,2, \ldots, L$ and $G_{n, m}^{(\mathcal{W})}=0$ otherwise when the OFDM signal is $\mathcal{W}_{L}$-based, and

$$
G_{n+\frac{N}{2^{u}} v, \chi(u)+n}^{(\mathcal{V})}=2^{-\frac{u}{2}} \phi_{u, v}, \quad n \in \mathcal{Z}_{N / 2^{u}} \text { and } v \in \mathcal{Z}_{2^{u}}
$$

for $u=1,2, \ldots, L$ and $G_{n, m}^{(\mathcal{V})}=0$ otherwise when the OFDM signal is $\mathcal{V}_{L}$-based. Apparently, (14) defines a precoded signal format in which each OFDM data block is linearly encoded by (15) prior to OFDM modulation. For convenience, we shall refer to the OFDM system as $\mathcal{W}_{L}$-coded and $\mathcal{V}_{L}$-coded if $G_{n, m}^{(\mathcal{X})}$ 's are specified by (16) and (17), respectively.

Due to phase continuity, higher spectral compactness than uncoded OFDM signal can be achieved by several precoded OFDM signals, including $\mathcal{W}_{L}$ and $\mathcal{V}_{L}$-coded NG-OFDM and ZP-OFDM, as well as $\mathcal{V}_{L}$-coded CP-OFDM with $T_{g}=2^{-\varpi} T_{d}$ and $\varpi \in\left\{1,2, \ldots, \log _{2} N-L\right\}$. However, this spectral prevalence is not warranted for the other precoded CP-OFDM signals without phase continuity, including all $\mathcal{W}_{L}$-coded CP-OFDM signals and the $\mathcal{V}_{L}$-coded CP-OFDM signals with $T_{g}=\varrho T_{d}$ for $\varrho \notin\left\{2^{-1}, 2^{-2}, \ldots, 2^{-\left(\log _{2} N-L\right)}\right\}$. To improve spectral compactness, phase-discontinuous CP-OFDM can be aided by the phase-rotation mechanism with

$$
B_{n, k}=\exp \left\{j \frac{n}{2} \omega_{d} T_{g}\right\} \sum_{m=0}^{M-1} G_{n, m}^{(\mathcal{X})} D_{m, k}, \quad n \in \mathcal{Z}_{N}
$$

As shown later, noticeable improvement can be achieved when the ratio $T_{g} / T_{d}$ is small.

The signal model in (14) is useful in realizing the real waveform. Because (14) consists of a linear combination of rectangularly pulsed $\omega_{d}$-spaced complex exponentials, it permits efficient realization by $N$-point IFFT in conjunction with spectral precoding. Fig. 1 depicts the spectrally precoded OFDM system. As shown, each data block $\left\{D_{m, k}\right\}_{m=0}^{M-1}$ is first precoded by (15) or (18). Its output block $\left\{B_{n, k}\right\}_{n=0}^{N-1}$ is then fed into the standard IFFT-based OFDM transmission process to generate the signal (14). Obviously, the spectrally precoded OFDM transmitter differs from the uncoded OFDM transmitter by the insertion of a precoder characterized by (15) or (18). As shown in Table I, the additional complexities required by $\mathcal{W}_{L}$ and $\mathcal{V}_{L}$ precoders are the same and grow linearly with $2 N(L+2 \tau)$. This complexity overhead is about the same as the $\mathcal{G}_{L+1}$ precoder in [15].

\section{A. Spectral Analysis}

Using (15) and (18), $s(t)$ in (14) can be rewritten as

$$
s(t)=\rho \sum_{m=0}^{M-1} \sum_{k} \Re\left\{D_{m, k} q_{m}(t-k T)\right\} .
$$

Here, $q_{m}(t)$ is defined by $q_{m}(t) \triangleq \sum_{n=0}^{N-1} g_{n, m}^{(\mathcal{X})} \exp \left\{j\left(\omega_{0}+\right.\right.$ $\left.\left.n \omega_{d}\right) t\right\} p(t)$, where $g_{n, m}^{(\mathcal{X})} \triangleq \vartheta_{n} G_{n, m}^{(\mathcal{X})}$ with $\vartheta_{n}=$ $\exp \left\{j(n / 2) \omega_{d} T_{g}\right\}$ for CP-OFDM with phase rotation, and $\vartheta_{n}=1$ otherwise. Apparently, $s(t)$ is a multiplexing of $M$ independent component signals with zero mean. Moreover, each component signal carries a memoryless data stream and is orthogonal to its own $l T$-shifted version. Thus, the PSD of $s(t)$ can be obtained from the multiplexing of $M$ component signals bearing $\left\{D_{m, k}\right\}_{m=0}^{M-1}$ for one specific $k$, say $k=0$, and given by [23, eq. 2.57] as

$$
\mathcal{S}(f)=\frac{\rho^{2}}{4 T} \sum_{m=0}^{M-1} E\left\{\left|D_{m, 0} \mathcal{F}\left\{q_{m}(t)\right\}+D_{m, 0}^{*} \mathcal{F}\left\{q_{m}^{*}(t)\right\}\right|^{2}\right\}
$$


TABLE I

Complexity Comparison Among Various EnCoding ANd DeCoding Algorithms for $\mathcal{G}_{L}, \mathcal{V}_{L}$, AND $\mathcal{W}_{L}$-CODED OFDMS AND ZF DEMODULATION ALGORITHM FOR UNCODED OFDM

\begin{tabular}{c||c|c}
\hline Algorithm & $\begin{array}{c}\text { Number of Real } \\
\text { Multiplications } / T\end{array}$ & $\begin{array}{c}\text { Number of Real } \\
\text { Additions } / T\end{array}$ \\
\hline \hline $\begin{array}{c}\mathcal{W}_{L} \text { and } \mathcal{V}_{L} \\
\text { Encoding }\end{array}$ & $O(2 N(L+2 \tau))$ & $O(2 N(L-1+\tau))$ \\
\hline $\mathcal{W}_{L}$ and $\mathcal{V}_{L}$ & $O\left(2 N\left(3+2 \tau-2^{-L}\right)\right)$ & $O\left(2 N\left(L+\tau+2^{-L}\right)\right)$ \\
ZF Decoding & $O(2 N(L+1+2 \varphi))$ & $O(2 N(L+\varphi))$ \\
\hline $\mathcal{G}_{L}$ Encoding & $O(4 N)$ & $O\left(N J^{L}(2 L+2)+2 N(1+\varphi)\right)$ \\
\hline $\mathcal{G}_{L}$ ML Decoding & $O\left(N J^{L}(2 L+6)+4 N(1+\varphi)\right)$ & $O(2 N)$ \\
\hline $\begin{array}{l}\text { ZF Demodulation } \\
\text { (uncoded OFDM) }\end{array}$ & & \\
\hline
\end{tabular}

All the constants, sign inversions, and decision thresholds that are independent of data and received symbols are not counted in complexity evaluation. For the ML decoding of $\mathcal{G}_{L}$-coded OFDM, the $J^{2}$-ary quadrature amplitude modulation (QAM) is adopted as the component modulation. For $\mathcal{W}_{L}$ and $\mathcal{V}_{L}$ algorithms, $\tau=1$ if CP-OFDM with phase rotation is considered, and $\tau=0$ otherwise. For $\mathcal{G}_{L}$ algorithms, $\varphi=1$ if CP-OFDM is considered, and $\varphi=0$ otherwise.

with $\mathcal{F}\{\cdot\}$ denoting the Fourier transform. Since $\omega_{0} T \gg 1$, $\mathcal{F}\left\{q_{m}(t)\right\}$ and $\mathcal{F}\left\{q_{m}^{*}(t)\right\}$ contain virtually disjoint spectral portions. This further simplifies (20) to

$$
\mathcal{S}(f)=\frac{\rho^{2}}{4 T} \sum_{m=0}^{M-1}\left\{\left|\mathcal{F}\left\{q_{m}(t)\right\}\right|^{2}+\left|\mathcal{F}\left\{q_{m}^{*}(t)\right\}\right|^{2}\right\} .
$$

It follows that the equivalent lowpass PSDs for CP-OFDM and ZP-OFDM are obtained as

$$
\begin{aligned}
& \mathcal{S}_{\mathrm{LP}}^{\mathrm{CP}}(f) \\
& =\frac{\rho^{2} T}{2} \sum_{m=0}^{M-1} \mid \sum_{n=0}^{N-1} g_{n, m}^{(\mathcal{X})} \exp \left\{-j \frac{n}{2} \omega_{d} T_{g}\right\} \\
& \quad \times\left.\operatorname{sinc}\left(\left(n-\frac{N-1}{2}\right) \frac{T}{T_{d}}-f T\right)\right|^{2} \\
& \mathcal{S}_{\mathrm{LP}}^{\mathrm{ZP}}(f) \\
& =\frac{\rho^{2} T_{d}^{2}}{2 T} \sum_{m=0}^{M-1}\left|\sum_{n=0}^{N-1} G_{n, m}^{(\mathcal{X})} \operatorname{sinc}\left(n-\frac{N-1}{2}-f T_{d}\right)\right|^{2}
\end{aligned}
$$

where $\operatorname{sinc}(x) \triangleq \sin (\pi x) /(\pi x)$. The average signal power $P$ is related to $\rho$ by $P=\left(\rho^{2} / 2\right) \sum_{m=0}^{M-1} \sum_{n=0}^{N-1} \sum_{l=0}^{N-1} g_{n, m}^{(\mathcal{X})}\left(g_{l, m}^{(\mathcal{X})}\right)^{*}$ $\exp \left\{j((l-n) / 2) \omega_{d} T_{g}\right\} \operatorname{sinc}\left((n-l)\left(T / T_{d}\right)\right)$ for CP-OFDM and $P=\left(\rho^{2} T_{d} / 2 T\right) \sum_{m=0}^{M-1} \sum_{n=0}^{N-1}\left|G_{n, m}^{(\mathcal{X})}\right|^{2}$ for ZP-OFDM. When $T=T_{d}$, both (22) and (23) simplify to the $\mathcal{S}_{\mathrm{LP}}^{\mathrm{NG}}(f)$ for NG-OFDM which has the exact form of (23) with $T_{d}$ replaced by $T$.

Using (22) and (23), the equivalent lowpass PSDs for $\mathcal{W}_{L}$ and $\mathcal{V}_{L}$-coded OFDMs can be obtained by setting up $G_{n, m}^{(\mathcal{X})}$, s appropriately. For example

$$
\begin{aligned}
\mathcal{S}_{\mathrm{LP}}^{\mathrm{ZP}}(f) & \\
= & \frac{P T_{d}}{2 \pi^{2} M} \cos ^{2}\left(\pi f T_{d}\right) \\
& \times\left\{\sum_{n=0}^{\frac{N}{2}-1}\left(f T_{d}+\frac{N-1}{2}-2 n\right)^{-2}\left(f T_{d}+\frac{N-3}{2}-2 n\right)^{-2}\right.
\end{aligned}
$$

$$
\begin{aligned}
+\sum_{u=2}^{L} 2^{1-u} \sum_{n=0}^{\frac{N}{2^{u}}-1} \mid \sum_{v=0}^{2^{u-2}-1} & {\left[\frac{2^{u-1}+1}{\kappa_{v}\left(\kappa_{v}-2^{u-1}-1\right)}\right.} \\
& \left.\left.+\frac{2^{u-1}-1}{\left(\kappa_{v}-1\right)\left(\kappa_{v}-2^{u-1}\right)}\right]\left.\right|^{2}\right\}
\end{aligned}
$$

for $\mathcal{W}_{L}-$ coded ZP-OFDM $\mathcal{S}_{\mathrm{LP}}^{\mathrm{ZP}}(f)$

$$
\begin{aligned}
= & \frac{N^{2} P T_{d}}{\pi^{2} M} \cos ^{2}\left(\pi f T_{d}\right) \\
& \times \sum_{u=1}^{L} 2^{-3 u} \sum_{n=0}^{\frac{N}{2^{u}}-1}\left|\sum_{v=0}^{2^{u-1}-1} \lambda_{v}^{-1}\left(\lambda_{v}-\frac{N}{2^{u}}\right)^{-1}\right|^{2} \\
& \quad \text { for } \mathcal{V}_{L}-\text { coded ZP-OFDM }
\end{aligned}
$$

where $\lambda_{v} \triangleq f T_{d}+((N-1) / 2)-n-\left(N / 2^{u-1}\right) v$ and $\kappa_{v} \triangleq f T_{d}+((N-1) / 2)-n 2^{u}-2 v$. As indicated by (24) and (25), power spectral sidelobes of $\mathcal{W}_{L}$ and $\mathcal{V}_{L}$-coded ZP-OFDMs fall off as $f^{-4}$, and much faster than those of uncoded ZP-OFDM, which falls as $f^{-2}$ [15]. This observation also holds for NG-OFDMs because their $\mathcal{S}_{\mathrm{LP}}^{\mathrm{NG}}(f)$ 's have the same forms as (24) and (25) with $T_{d}=T$.

When $\varpi \in\left\{1,2, \ldots, \log _{2} N-L\right\}$, the equivalent lowpass PSD for $\mathcal{V}_{L}$-coded CP-OFDM with $T_{g}=2^{-\varpi} T_{d}$ and without phase rotation is given by

$$
\begin{aligned}
\mathcal{S}_{\mathrm{LP}}^{\mathrm{CP}}(f)= & \frac{P T}{\pi^{2} M} \sum_{u=1}^{L} 2^{-u} \xi_{u}^{2} \\
& \times \sum_{n=0}^{\frac{N}{2^{u}}-1} \sin ^{2}\left(\pi \epsilon_{n}\right)\left|\sum_{v=0}^{2^{u-1}-1} \delta_{v}^{-1}\left(\delta_{v}-\xi_{u}\right)^{-1}\right|^{2}
\end{aligned}
$$

with $\epsilon_{n} \triangleq f T+(((N-1) / 2)-n)\left(1+2^{-\varpi}\right), \xi_{u} \triangleq$ $N 2^{-u}\left(1+2^{-\varpi}\right)$, and $\delta_{v} \triangleq \epsilon_{n}-\left(N / 2^{u-1}\right) v\left(1+2^{-\varpi}\right)$. Obviously, power spectral sidelobes fall off as $f^{-4}$ for such $\mathcal{V}_{L}$-coded CP-OFDMs. 


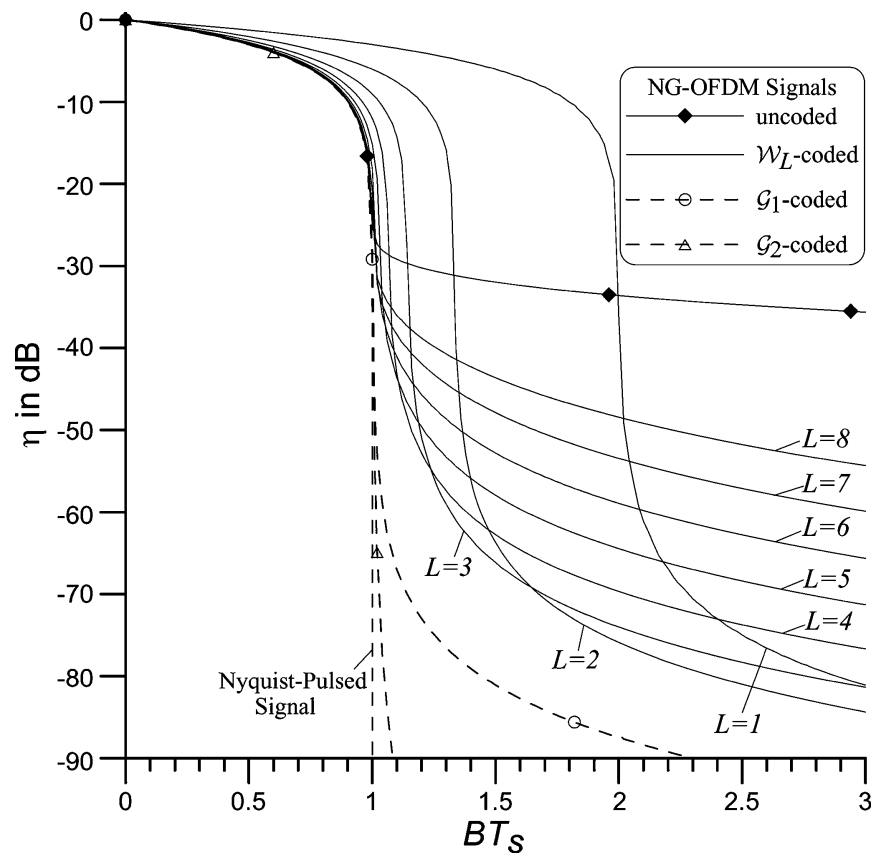

Fig. 2. Fractional out-of-band power characteristics of $\mathcal{W}_{L}$-coded NG-OFDM signals with $N=256$.

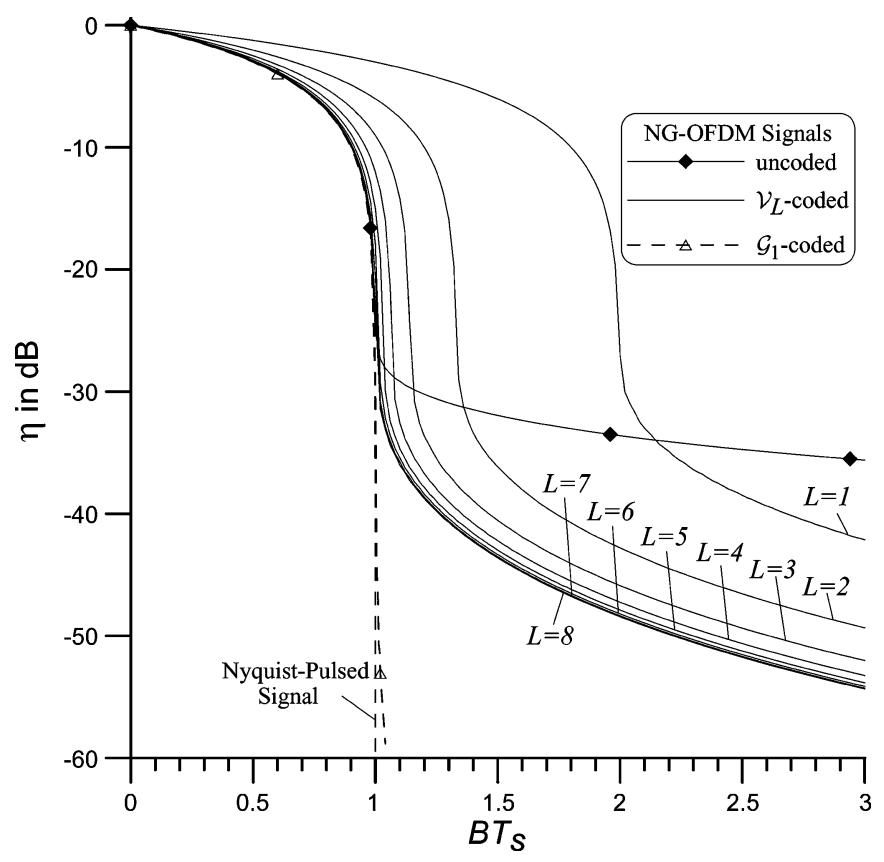

Fig. 3. Fractional out-of-band power characteristics of $\mathcal{V}_{L}$-coded NG-OFDM signals with $N=256$.

\section{B. Spectral Trends}

In what follows, the spectral compactness is characterized by the fractional out-of-band power

$$
\eta=10 \log _{10}\left(1-\frac{1}{P} \int_{-B / 2}^{B / 2} \mathcal{S}_{\mathrm{LP}}(f) d f\right)
$$

which denotes the fraction of total power that is not captured within the frequency band $[-B / 2, B / 2]$. In particular, the spectral trends on $\eta$ for various signals are studied with respect to

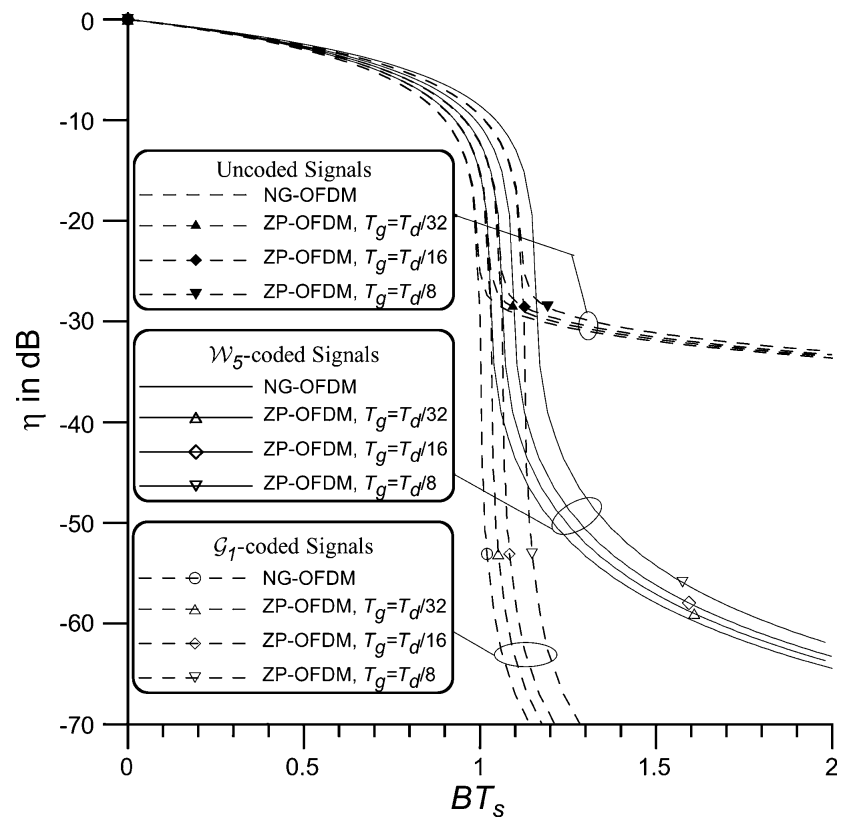

Fig. 4. Fractional out-of-band power characteristics of $\mathcal{W}_{5}$-coded ZP-OFDM signals with $N=256$

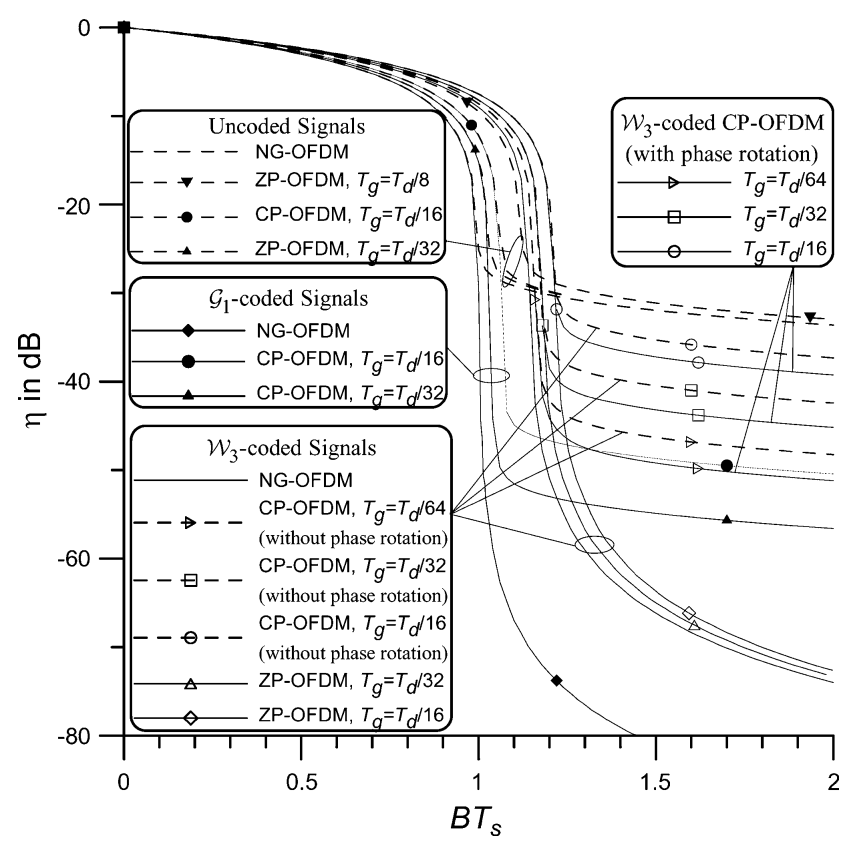

Fig. 5. Fractional out-of-band power characteristics of $\mathcal{W}_{3}$-coded CP-OFDM and ZP-OFDM signals with $N=256$.

the normalized bandwidth $B T_{s}$ so that the spectral efficiency can be compared among different signals with the same data symbol rate. Here, the spectral efficiency is referred to as the inverse of $B T_{s}$ that is required to achieve a given $\eta$. Therefore, to achieve a fixed $\eta$, the signal requiring a smaller $B T_{s}$ can exhibit higher spectral efficiency.

Figs. 2-7 illustrate the spectral performance trends achieved by precoded OFDM versus (uncoded) rectangularly pulsed OFDM and correlatively $\mathcal{G}_{L^{\prime}}$-coded OFDM. Although presented for $N=256$, the demonstrated trends hold generally for other admissible $N$ values.

Fig. 2 illustrates the spectral efficiencies of $\mathcal{W}_{L}$-coded NG-OFDM signals. The ideal performance of the single-carrier 


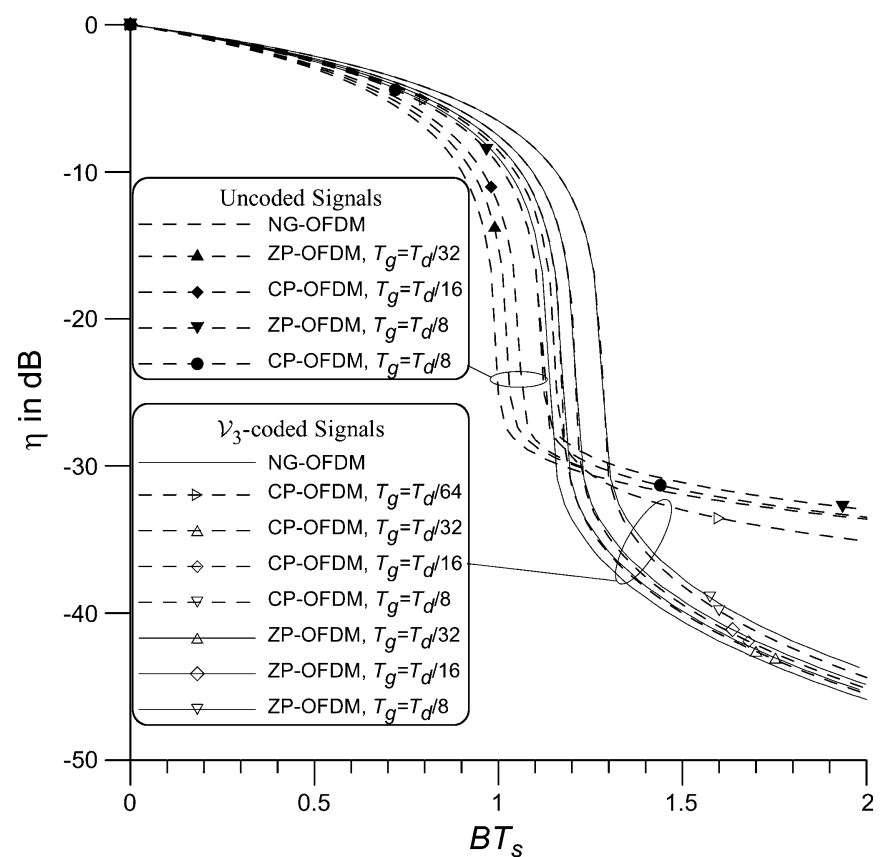

Fig. 6. Fractional out-of-band power characteristics of $\mathcal{V}_{3}$-coded CP-OFDM and ZP-OFDM signals with $N=256$.

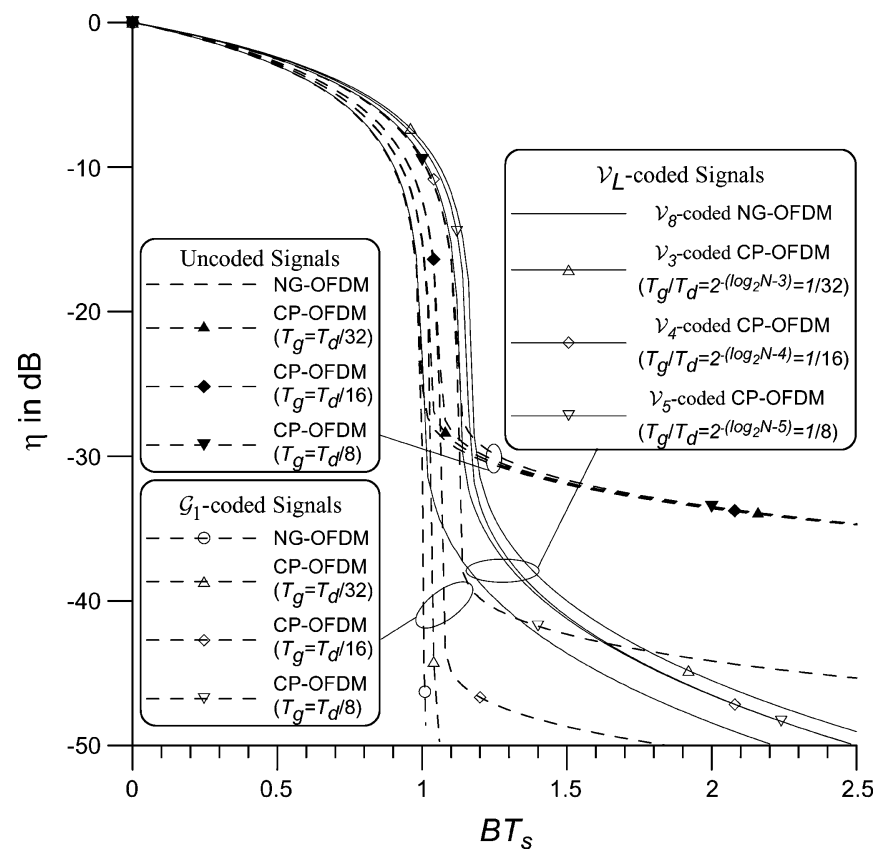

Fig. 7. Fractional out-of-band power characteristics of $\mathcal{V}_{L}$-coded CP-OFDM signals with $N=256$.

Nyquist-pulsed signal with $\mathcal{S}_{L P}(f)=T_{s}$ if $|f|<1 /\left(2 T_{s}\right)$ and $\mathcal{S}_{L P}(f)=0$ otherwise is also plotted as a benchmark. Note that $\mathcal{W}_{1}$-coded NG-OFDM corresponds to NG-OFDM based on the $N$-D $\Omega_{4}(1)$ in [21]. As shown, $\mathcal{W}_{L}$-coded NG-OFDM with $L>1$ significantly outperforms, in spectral efficiency, the conventional $\mathcal{W}_{1}$-coded NG-OFDM when the required $\eta$ is not extremely small. When the required $\eta$ is very small (say, $-50 \mathrm{~dB}$ or less), very large improvement over uncoded NG-OFDM is achieved by $\mathcal{W}_{L}$-coded NG-OFDM with a medium $L$. Moreover, $\mathcal{W}_{L}$-coded NG-OFDM with a medium $L$ (say, $L=5$ ) can perform closer to the Nyquist-pulsed signal and $\mathcal{G}_{L^{\prime}}$-coded OFDM signals for small $\eta$ values than uncoded NG-OFDM. The spectral efficiencies of $\mathcal{V}_{L}$-coded NG-OFDM signals are similarly compared in Fig. 3. As indicated, the spectral efficiency of $\mathcal{V}_{L}$-coded NG-OFDM improves over uncoded NG-OFDM when the required $\eta$ is small, and more improvement can be achieved when a larger $L$ is used. Comparing Figs. 2 and 3 shows that $\mathcal{V}_{L}$-coded NG-OFDM is generally outperformed by $\mathcal{W}_{L^{\prime}}$-coded NG-OFDM with a medium $L^{\prime}$ when the required $\eta$ is very small (say, $-50 \mathrm{~dB}$ or less). We also note that correlatively $\mathcal{G}_{L^{\prime}}$-coded OFDM with a small $L^{\prime}$ can provide the spectral compactness very close to the Nyquist-pulsed signal. This prevalence is, however, achieved at the cost of remarkable degradation in error performance from uncoded OFDM on AWGN and some DS channels [15].

Observe (23) for ZP-OFDM. When the same $G_{n, m}^{(\mathcal{X})}$, s, $M, N$, and $T$ are used, the $\mathcal{S}_{\mathrm{LP}, 1}^{\mathrm{ZP}}(f)$ of ZP-OFDM using $T_{d, 1}$ is related to the $\mathcal{S}_{\mathrm{LP}, 2}^{\mathrm{ZP}}(f)$ of ZP-OFDM using $T_{d, 2}$ by $\mathcal{S}_{\mathrm{LP}, 1}^{\mathrm{ZP}}\left(f / T_{d, 1}\right) / T_{d, 1}=\mathcal{S}_{\mathrm{LP}, 2}^{\mathrm{ZP}}\left(f / T_{d, 2}\right) / T_{d, 2}$. Using this in (27) reveals that the ZP-OFDM signal using $T_{d}$ exhibits $\left(T_{d} / T\right)$-fold the spectral efficiency of the NG-OFDM signal, provided that both signals are based on the same $G_{n, m}^{(\mathcal{X})}$ 's, $M, N$, and $T$. Thus, the results in Figs. 2 and 3 also apply to ZP-OFDM with the spectral efficiencies scaled down by a factor $T_{d} / T$. It follows

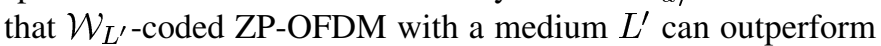
$\mathcal{V}_{L}$-coded ZP-OFDM in spectral efficiency when the required $\eta$ is very small. Hence, $\mathcal{W}_{L}$ coding turns out to be very suited for use in ZP-OFDM. Fig. 4 illustrates the spectral efficiencies of $\mathcal{W}_{5}$-coded ZP-OFDM for various $T_{g} / T_{d}$ ratios. As shown, ZP-OFDM degrades more severely from NG-OFDM when a larger $T_{g} / T_{d}$ is used. Using the same $T_{g} / T_{d}$ ratio, $\mathcal{W}_{5}$-coded ZP-OFDM is shown to outperform uncoded ZP-OFDM significantly in spectral efficiency when the required $\eta$ is small (say, $-40 \mathrm{~dB}$ or less), and provide the spectral efficiency closer to $\mathcal{G}_{1}$-coded ZP-OFDM for small $\eta$ values than uncoded ZP-OFDM.

Like ZP-OFDM, CP-OFDM degrades from NG-OFDM in spectral efficiency due to the use of guard intervals. This phenomenon is illustrated in Figs. 5 and 6 , where $\mathcal{W}_{3}$-coded and $\mathcal{V}_{3}$-coded CP-OFDM signals with various $T_{g} / T_{d}$ ratios are compared with the corresponding NG-OFDM and ZP-OFDM signals. In Fig. 5, $\mathcal{W}_{3}$-coded CP-OFDM is shown to degrade severely from $\mathcal{W}_{3}$-coded ZP-OFDM with the same $T_{g} / T_{d}$, especially when $T_{g} / T_{d}$ is large. This is primarily due to the deficiency of phase continuity in the $\mathcal{W}_{L}$-coded CP-OFDM signaling. The same performance trend also exists with $\mathcal{G}_{L^{\prime}}$-coded CP-OFDM versus $\mathcal{G}_{L^{\prime}}$-coded ZP-OFDM [15]. As also indicated, $\mathcal{W}_{3}$-coded CP-OFDM with phase rotation performs better than $\mathcal{W}_{3}$-coded CP-OFDM without phase rotation, especially when $T_{g} / T_{d}$ is small, but still worse than $\mathcal{G}_{1}$-coded CP-OFDM. Nevertheless, when the required $\eta$ is small (say, $-40 \mathrm{~dB}$ ), $\mathcal{W}_{3}$-coded CP-OFDM with phase rotation can provide much higher spectral efficiency than uncoded CP-OFDM if $T_{g} / T_{d}$ is small (say, 1/32). Because the continuous-phase attribute is still sustained, the $\mathcal{V}_{3}$-coded CP-OFDM signals with $T_{g} / T_{d} \in\left\{2^{-3}, 2^{-4}, 2^{-5}\right\}$ are found in Fig. 6 to perform very close to the corresponding $\mathcal{V}_{3}$-coded ZP-OFDM signals. 
However, $\mathcal{V}_{3}$-coded CP-OFDM with $T_{g} / T_{d}=2^{-6}$ degrades severely due to the loss of phase continuity. This clearly illustrates the advantage of using $\mathcal{V}_{L}$ coding for CP-OFDM with $T_{g} / T_{d}$ constrained in the specific set $\left\{2^{-1}, 2^{-2}, \ldots, 2^{-\left(\log _{2} N-L\right)}\right\}$. As highlighted by the performance trend that $\mathcal{V}_{L}$-coded ZP-OFDM performs better when a larger $L$ is used, it is thus natural to adopt $\mathcal{V}_{L}$-coded CP-OFDM when the guard ratio is fixed by $T_{q} / T_{d}=2^{-\left(\log _{2} N-L\right)}$. As illustrated in Fig. 7, $\mathcal{V}_{L}$-coded CP-OFDM with $T_{g} / T_{d}=2^{-\left(\log _{2} N-L\right)}$ can provide much higher spectral efficiency, in relative to a small required $\eta$ (say, $-40 \mathrm{~dB}$ or less), than uncoded OFDM and, in some case (e.g., $T_{g} / T_{d}=2^{-3}$ ), $\mathcal{G}_{1}$-coded CP-OFDM.

\section{ZF DEMODULATION}

When the channel response is available or can be perfectly measured, the ZF demodulation technique is commonly used in bandlimited communications to counteract the channel effect and then enable data detection based on the minimum Euclidean distance principle [23]. This technique is adopted here to demodulate the spectrally coded OFDM signals, assuming that the channel response is known a priori.

To facilitate the ZF demodulation, it is essential to develop the methods of decoding $\left\{B_{n, k}\right\}_{n=0}^{N-1}$ back to $\left\{D_{m, k}\right\}_{m=0}^{M-1}$, provided that $B_{n, k}$ 's are specified by (15) or (18) for $\mathcal{W}_{L}$ and $\mathcal{V}_{L}$ codes. The methods are derived in Appendices D and E and given as follows.

Proposition 3: When $B_{n, k}$ s are $\mathcal{W}_{L}$-coded, $D_{m, k}$ 's can be obtained by

$$
\begin{aligned}
D_{\chi(u)+n, k}=\sum_{v=0}^{2^{u}-1} \vartheta_{n 2^{u}+v}^{*} G_{n 2^{u}+v, \chi(u)+n}^{(\mathcal{W})} B_{n 2^{u}+v, k}, \\
\quad \text { for } n \in \mathcal{Z}_{N / 2^{u}} \text { and } u=1,2, \ldots, L .
\end{aligned}
$$

Proposition 4: When $B_{n, k}$ 's are $\mathcal{V}_{L}$-coded, $D_{m, k}$ 's can be obtained by

$$
\begin{aligned}
D_{\chi(u)+n, k}=\sum_{v=0}^{2^{u}-1} \vartheta_{n+\frac{N}{2^{u}} v}^{*} G_{n+\frac{N}{2^{u}} v, \chi(u)+n}^{(\mathcal{V})} B_{n+\frac{N}{2^{u}} v, k}, \\
\text { for } n \in \mathcal{Z}_{N / 2^{u}} \text { and } u=1,2, \ldots, L .
\end{aligned}
$$

Assuming perfect alignment in timing and channel response at the receiver, the spectrally precoded OFDM signals can be coherently demodulated as in Fig. 1. The received waveform is first manipulated by the standard FFT-based OFDM receiving process to yield each useful block $\left\{R_{n, k}\right\}_{n=0}^{N-1}$, with $R_{n, k}=$ $H_{n} B_{n, k}+Y_{n, k}$ representing the received complex symbol on the $n$th subcarrier for the $k$ th signaling interval. Here, $H_{n}$ is the complex channel response on the $n$th subcarrier, and $Y_{n, k}$ 's are complex AWGN samples which are i.i.d. with zero mean and identical variance $\sigma_{Y}^{2}$. The ZF decoder is then used to recover $\left\{D_{m, k}\right\}_{m=0}^{M-1}$ by processing $\left\{R_{n, k}\right\}_{n=0}^{N-1}$. Specifically, the decoding rule is to find the candidate data symbol $\widehat{D}_{m, k}$ that is closest in Euclidean distance to $X_{m, k}$ for each $m \in \mathcal{Z}_{M}$, where $X_{m, k}$ is the test metric defined by

$$
\begin{gathered}
X_{\chi(u)+n, k}=\sum_{v=0}^{2^{u}-1} \vartheta_{n 2^{u}+v}^{*} G_{n 2^{u}+v, \chi(u)+n}^{(\mathcal{W})} \frac{R_{n 2^{u}+v, k}}{H_{n 2^{u}+v}}, \\
n \in \mathcal{Z}_{N / 2^{u}} \text { and } u=1,2, \ldots, L
\end{gathered}
$$

$$
\begin{gathered}
X_{\chi(u)+n, k}=\sum_{v=0}^{2^{u}-1} \vartheta_{n+\frac{N}{2^{u}} v}^{*} G_{n+\frac{N}{2^{u}} v, \chi(u)+n}^{(\mathcal{V})} \frac{R_{n+\frac{N}{2^{u}} v, k}}{H_{n+\frac{N}{2^{u}} v}}, \\
n \in \mathcal{Z}_{N / 2^{u}} \text { and } u=1,2, \ldots, L
\end{gathered}
$$

for $\mathcal{W}_{L}$ and $\mathcal{V}_{L}$-coded OFDM, respectively. As shown in Propositions 3 and 4 , this rule can correctly decode $\widehat{D}_{m, k}=D_{m, k}$ for $m \in \mathcal{Z}_{M}$ if the noise samples $Y_{n, k}$ 's are absent. In the presence of noise, both (30) and (31) can be equivalently modeled as $X_{\chi(u)+n, k}=D_{\chi(u)+n, k}+U_{\chi(u)+n, k}$, where we have defined

$$
U_{\chi(u)+n, k} \triangleq \sum_{v=0}^{2^{u}-1} \vartheta_{n 2^{u}+v}^{*} G_{n 2^{u}+v, \chi(u)+n}^{(\mathcal{W})} Y_{n 2^{u}+v, k} / H_{n 2^{u}+v}
$$

for $\mathcal{W}_{L}$-coded OFDM and

$$
\begin{gathered}
U_{\chi(u)+n, k} \triangleq \sum_{v=0}^{2^{u}-1} \vartheta_{n+\left(N / 2^{u}\right) v}^{*} G_{n+\left(N / 2^{u}\right) v, \chi(u)+n}^{(\mathcal{V})} \\
Y_{n+\left(N / 2^{u}\right) v, k} / H_{n+\left(N / 2^{u}\right) v}
\end{gathered}
$$

for $\mathcal{V}_{L}$-coded OFDM. In both cases, $U_{\chi(u)+n, k}$ 's are circularly symmetric complex Gaussian noise samples with zero mean, and the equivalent model is exactly the same as the subcarrier demodulation model for uncoded OFDM with perfect channel estimation and ZF demodulation [1]-[3].

Therefore, when the same component modulation is used, the error probabilities of subcarrier demodulation for uncoded and spectrally precoded OFDM are given by the same function which is inversely proportional to $\gamma_{s, \text { unc }}(l)$ and $\gamma_{s, \text { cod }}(u, n)$, respectively. Here, $\gamma_{s, \text { unc }}(l) \triangleq \sigma_{Y}^{-2}\left|H_{l}\right|^{2}$ is the signal-to-noise power ratio (SNR) on the lth subcarrier for uncoded OFDM, and $\gamma_{s, \operatorname{cod}}(u, n)$ is the SNR on channel $\chi(u)+n$ for $\mathcal{W}_{L}$ and $\mathcal{V}_{L}$-coded OFDMs defined by $\gamma_{s, \operatorname{cod}}(u, n) \triangleq \sigma_{Y}^{-2} 2^{u}\left(\sum_{v=0}^{2^{u}-1}\left|H_{n 2^{u}+v}\right|^{-2}\right)^{-1}$ for $\mathcal{W}_{L}$-coded OFDM and $\gamma_{s, \operatorname{cod}}(u, n) \triangleq \sigma_{Y}^{-2} 2^{u}\left(\sum_{v=0}^{2^{u}-1}\left|H_{n+\left(N / 2^{u}\right) v}\right|^{-2}\right)^{-1}$ for $\mathcal{V}_{L}$-coded OFDM. When $H_{l}$ 's have constant magnitudes (e.g., AWGN channel), $\gamma_{s, \text { unc }}(l)$ and $\gamma_{s, \text { cod }}(u, n)$ are equal for all indices and, thus, $\mathcal{W}_{L}$ and $\mathcal{V}_{L}$-coded OFDMs perform the same as uncoded OFDM. When $H_{l}$ 's have nonconstant magnitudes (e.g., delay spread channels), the inequality $\max _{l} \gamma_{s, \text { unc }}(l) \geq \gamma_{s, \operatorname{cod}}(u, n) \geq \min _{l} \gamma_{s, \text { unc }}(l)$ holds for all $u$ and $n$ and for both $\mathcal{W}_{L}$ and $\mathcal{V}_{L}$-coded OFDMs, due to the averaging nature in $\gamma_{s, \operatorname{cod}}(u, n)$. This implies that $\mathcal{W}_{L}$ and $\mathcal{V}_{L}$-coded OFDMs perform comparably with uncoded OFDM when the average bit-error rate (ABER) is concerned. Note also that $\mathcal{W}_{L}$-coded OFDM combines signal powers over adjacent subcarriers, while $\mathcal{V}_{L}$-coded OFDM over distant subcarriers. This further implicates that $\mathcal{W}_{L}$ and $\mathcal{V}_{L}$-coded OFDMs perform very differently on DS channels with subcarrier amplitude fluctuation.

Fig. 8 illustrates the comparison of ABER results among various CP-OFDM ZF demodulation schemes with Gray-labeled 16-ary QAM component modulations on AWGN and four DS channels. The CP length is assumed long enough to reject the intersymbol interference, and $E_{b} / N_{0}$ is defined as the bit SNR for useful data blocks. The results of uncoded, $\mathcal{V}_{3}$-coded, and $\mathcal{W}_{3}$-coded CP-OFDM schemes are obtained by using the analytical expression in [23, eq. 10.36a]. The results of $\mathcal{G}_{L}$-coded CP-OFDM schemes are obtained by simulating 


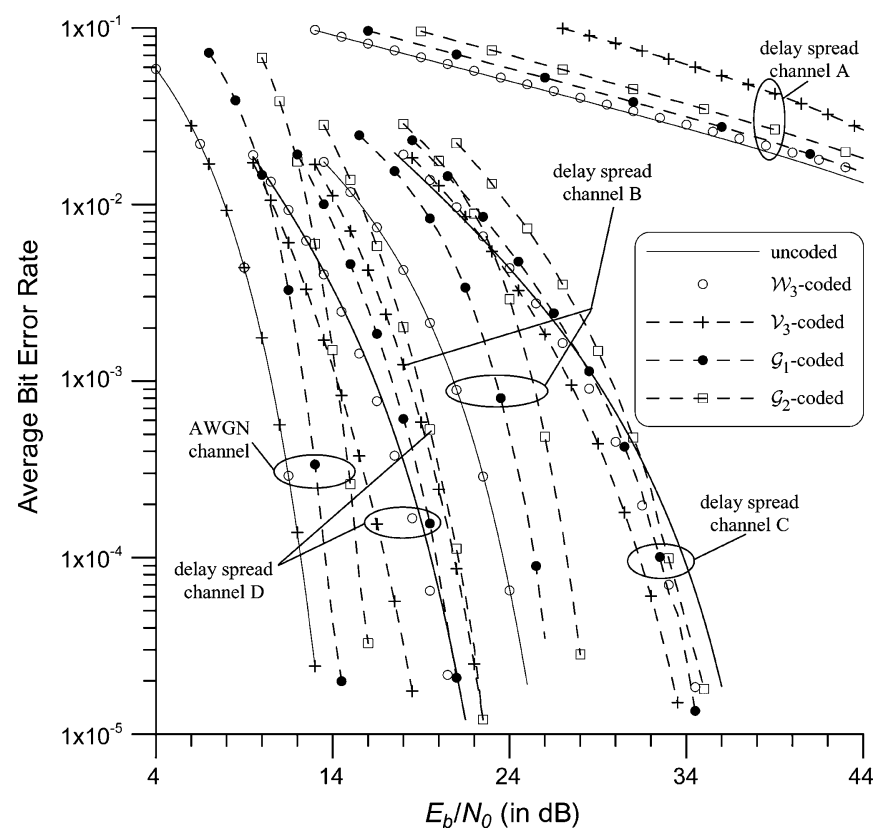

Fig. 8. ABER characteristics of Gray-labeled CP-OFDM with $N=256$ and 16-ary QAM component modulation.

the maximum-likelihood (ML) block decoding rule in [15]. The DS channels are defined by $H_{l}=\left.H(z)\right|_{z=\exp \{j 2 l \pi / N\}}$ with $H(z)=0.407+0.815 z^{-1}+0.407 z^{-2}, H(z)=0.8+0.6 z^{-1}$, $H(z)=0.8\left(0.8+0.6 z^{-1}\right)+0.6\left(0.8 z^{-16}+0.6 z^{-17}\right)$, and $H(z)=0.895+0.36 z^{-5}+0.263 z^{-13}$ for Channels $\mathrm{A}, \mathrm{B}$, $\mathrm{C}$, and D, respectively. Channels A and B are typified in [6] and [15] as the DS channels with continuous spreads, where Channel A is a spectral-null channel and Channel B has no spectral null but small channel amplitudes at some subcarriers. Channels C and D represent the DS channels that have dispersive spreads but no spectral null, and thus characterized by more fluctuation on subcarrier amplitudes than Channels $\mathrm{A}$ and B. As shown, uncoded, $\mathcal{V}_{3}$-coded, and $\mathcal{W}_{3}$-coded schemes perform the same, and outperform $\mathcal{G}_{L^{\prime}}$-coded schemes remarkably on the AWGN channel. The performance improvement over the $\mathcal{G}_{L^{\prime}}$-coded scheme is larger as $L^{\prime}$ is increased. Such performance prevalence over $\mathcal{G}_{L^{\prime}}$-coded schemes comes from the difference in signaling structure that the orthogonality among multiplexed data-modulated waveforms on useful data intervals is sustained in $\mathcal{W}_{L}$ and $\mathcal{V}_{L}$-coded schemes [see (12) and (13)], but broken in $\mathcal{G}_{L^{\prime}}$-coded schemes [15]. However, the performance characteristics among various schemes differ on DS channels. On Channels A and B that have continuous spreads, $\mathcal{W}_{3}$-coded scheme performs almost the same as the uncoded scheme, and remarkably better than $\mathcal{G}_{L^{\prime}}$-coded schemes. Nevertheless, this performance advantage diminishes, especially in low ABERs, on Channels $\mathrm{C}$ and $\mathrm{D}$ that have dispersive spreads. As thus indicated, the $\mathcal{G}_{L^{\prime}}$-coded scheme that makes decisions over the entire OFDM block is more robust to high-amplitude fluctuation inherent in Channels $\mathrm{C}$ and $\mathrm{D}$ than the $\mathcal{W}_{L}$-coded scheme that combines signal powers over adjacent subcarriers. By combining signal powers over distant subcarriers, $\mathcal{V}_{3}$-coded scheme is also shown in the figure to provide the best performance (in medium and low ABERs) on all DS channels without spectral null, but the worst performance (in high ABERs) on the DS channel with spectral null.

In Table I, the complexities required for digitally realizing various demodulation schemes are given. As indicated, the complexity for $\mathcal{W}_{L}$ and $\mathcal{V}_{L} \mathrm{ZF}$ decoding grows linearly with $2 N(3+$ $2 \tau)$ and is about double the complexity for uncoded OFDM demodulation. Moreover, $\mathcal{W}_{L}$ and $\mathcal{V}_{L} \mathrm{ZF}$ decoding requires much less complexity than $\mathcal{G}_{L^{\prime}}$ ML decoding.

To enable $\mathrm{ZF}$ demodulation, accurate estimation on the channel impulse response is required at the receiver. Such channel estimation can be achieved with the aid of decision-feedback symbols at the receiver or predetermined pilot symbols at the transmitter. Due to $\mathcal{W}_{L}$ and $\mathcal{V}_{L}$ precoding, decision-feedback or pilot symbols need to be spectrally encoded before invoking conventional estimation algorithms (e.g., [30]) at the receiver. As indicated in Table I, this encoding process requires additional complexity that grows linearly with $2 N_{\mathrm{CE}}(L+2 \tau)$, in which $N_{\mathrm{CE}}$ is the number of subcarrier channels to be estimated. In the case that pilot symbols are scattered in time and frequency, the encoding operations in (45) and (52) can be used to allocate the spectrally encoded pilot symbols. For example, the scattered $\mathcal{V}_{L}$-encoded pilot symbols can be allocated as follows. By decomposing a number $\nu$ in $\mathcal{Z}_{N}$ as $\nu=\sum_{l=1}^{L} \nu_{l}\left(N / 2^{l}\right)+\nu_{L+1}$ with $\nu_{L+1} \in \mathcal{Z}_{N / 2^{L}}$ and $\nu_{l} \in \mathcal{Z}_{2}$ for $l \in\{1,2, \ldots, L\}$, the set $\mathcal{B}_{k}\left(\nu_{2}, \nu_{3}, \ldots, \nu_{L+1}\right) \triangleq\left\{B_{\nu, k} ; \nu_{1} \in \mathcal{Z}_{2}\right\}$ is shown in (52) to be determined by $\mathcal{D}_{k}\left(\nu_{2}, \nu_{3}, \ldots, \nu_{L+1}\right) \triangleq$ $\left\{D_{\chi(l)+\sum_{m=l+1}^{L} \nu_{m}\left(N / 2^{m}\right)+\nu_{L+1}, k} ; l \in\{1,2, \ldots, L\}\right\}$. When a subset $\mathcal{A}$ in $\overline{\mathcal{Z}}_{N / 2}$ is specified, the set of scattered $\mathcal{V}_{L}$-encoded pilot symbols $\left\{\mathcal{B}_{k}\left(\nu_{2}, \nu_{3}, \ldots, \nu_{L+1}\right) ; \sum_{l=2}^{L} \nu_{l}\left(N / 2^{l}\right)+\nu_{L+1} \in\right.$ $\mathcal{A}\}$ can be generated via (52) with predetermined symbols assigned to $\left\{\mathcal{D}_{k}\left(\nu_{2}, \nu_{3}, \ldots, \nu_{L+1}\right) ; \sum_{l=2}^{L} \nu_{l}\left(N / 2^{l}\right)+\nu_{L+1} \in\right.$ $\mathcal{A}\}$. Note that $\mathcal{A}$ allocates the subcarriers carrying pilot symbols and can be flexibly adapted.

\section{CONCLUSION}

Several spectrally precoded OFDM signaling formats which allow for FFT realization and guard insertion are proposed to improve the spectral compactness of rectangularly pulsed OFDM without trading off error performance. Particularly, the spectrally precoded OFDM signals have continuous phase and are analytically shown to exhibit relatively small power spectral sidelobes which fall off as $f^{-4}$. The ZF receiver that permits FFT realization is also developed to demodulate the spectrally precoded OFDM signals coherently. When the channel response is perfectly available, the proposed spectrally precoded OFDM schemes are shown to prevail in average error-rate characteristics over uncoded and correlatively coded rectangularly-pulsed OFDM for various DS channels.

\section{APPENDIX}

\section{A. Useful Identities}

By trigonometric identities, we have

$$
\frac{1}{\sqrt{2}}\left[C_{a}^{(\alpha)}(t)+C_{b}^{(\alpha)}(t)\right]=P_{b-a}^{(c)}(t) C_{\lfloor(a+b+\alpha) / 2\rfloor}^{(\beta)}(t)
$$




$$
\begin{aligned}
\frac{1}{\sqrt{2}}\left[C_{a}^{(\alpha)}(t)-C_{b}^{(\alpha)}(t)\right] & =P_{b-a}^{(s)}(t) S_{\lfloor(a+b+\alpha) / 2\rfloor}^{(\beta)}(t) \\
\frac{1}{\sqrt{2}}\left[S_{a}^{(\alpha)}(t)+S_{b}^{(\alpha)}(t)\right] & =P_{b-a}^{(c)}(t) S_{\lfloor(a+b+\alpha) / 2\rfloor}^{(\beta)}(t) \\
\left.\frac{1}{\sqrt{2}}\left[-S_{a}^{(\alpha)}(t)+S_{b}^{(\alpha)} t\right)\right] & =P_{b-a}^{(s)}(t) C_{\lfloor(a+b+\alpha) / 2\rfloor}^{(\beta)}(t)
\end{aligned}
$$

for integers $a, b, \alpha$, and $\beta$, where $\lfloor x\rfloor$ denotes the largest integer that is not larger than $x$ and the factor $\beta$ is given by $\beta=0$ if $\lfloor(a+b+\alpha) / 2\rfloor=(a+b+\alpha) / 2$ and $\beta=1$ otherwise.

\section{B. Derivation of (10)}

Quoting (32) and (34), we can easily obtain (10) with $u=1$ from (3). Next, consider $u=2,3, \ldots, \log _{2} N$. By invoking (35) once [giving (36)], then (34) $u-2$ times [giving (37)], and finally (33) once [giving (38)], we have from (4) that

$$
\begin{aligned}
P_{1}^{(s)}(t) & \left(\prod_{k=1}^{u-2} P_{2^{k}}^{(c)}(t)\right) P_{2^{u-1}}^{(s)}(t) C_{n 2^{u}+\zeta(u)}^{(1)}(t) \\
= & P_{1}^{(s)}(t)\left(\prod_{k=1}^{u-2} P_{2^{k}}^{(c)}(t)\right) \\
& \cdot\left(-2^{-\frac{1}{2}}\right) \sum_{l_{1}=0}^{1}(-1)^{l_{1}} S_{n 2^{u}+\zeta(u)-(-1)^{l_{1} 2^{u-2}}(1)}^{(1)}(t) \\
= & P_{1}^{(s)}(t) \cdot\left(-2^{-\frac{u-1}{2}}\right) \sum_{l_{1}, l_{2}, \ldots, l_{u-1}=0}^{1}(-1)^{l_{1}} \\
& \times S_{n 2^{u}+\zeta(u)-\sum_{i=1}^{u-1}(-1)^{l_{i}} 2^{u-i-1}}^{(1)}(t) \\
= & -2^{-\frac{u}{2}} \sum_{l_{1}, l_{2}, \ldots, l_{u}=0}^{1}(-1)^{l_{1}+l_{u}} C_{n 2^{u}+\sum_{i=1}^{u} l_{i} 2^{u-i}}^{(0)}(t) .
\end{aligned}
$$

Similarly, by invoking (33) once, then (32) $u-2$ times, and finally (35) once, we have

$$
\begin{aligned}
P_{1}^{(s)}(t) & \left(\prod_{k=1}^{u-2} P_{2^{k}}^{(c)}(t)\right) P_{2^{u-1}}^{(s)}(t) S_{n 2^{u}+\zeta(u)}^{(1)}(t) \\
= & -2^{-\frac{u}{2}} \sum_{l_{1}, l_{2}, \ldots, l_{u}=0}^{1}(-1)^{l_{1}+l_{u}} S_{n 2^{u}+\sum_{i=1}^{u} l_{i} 2^{u-i}}^{(0)}(t) .
\end{aligned}
$$

If $\left(l_{1}, l_{2}, \ldots, l_{u}\right)$ is used to denote the binary representation of the modulo- $2^{u}$ value of an integer $v,(38)$ and (39) can be translated to yield (10) for $\Phi_{u}^{(c)}$ with $u \in\left\{2,3, \ldots, \log _{2} N\right\}$.

\section{Derivation of (11)}

First, consider $u=1,2, \ldots, \log _{2} N-1$. By invoking (35) once [giving (40)] and then (34) $u-1$ times [giving (41)], we have from (6) that

$$
\begin{aligned}
& \left(\prod_{k=1}^{u-1} P_{\frac{N}{2^{k}}}^{(c)}(t)\right) P_{\frac{N}{2^{u}}}^{(s)}(t) C_{n+\frac{1}{2} \chi(u+1)}^{(0)}(t) \\
& =-\left(\prod_{k=1}^{u-1} P_{\frac{N}{2^{k}}}^{(c)}(t)\right) 2^{-\frac{1}{2}} \sum_{l_{1}=0}^{1}(-1)^{l_{1}} S_{n+\frac{1}{2} \chi(u)+\frac{N}{2^{u}} l_{1}}^{(0)}(t) \\
& =-2^{-\frac{u}{2}} \sum_{l_{1}, l_{2}, \ldots, l_{u}=0}^{1}(-1)^{l_{1}} S_{n+N}^{(0)} \sum_{k=1}^{u} l_{k} 2^{k-u-1}
\end{aligned}
$$

Similarly, by invoking (33) once and then (32) $u-1$ times, we have from (6) that

$$
\begin{aligned}
& \left(\prod_{k=1}^{u-1} P_{\frac{N}{2^{k}}}^{(c)}(t)\right) P_{\frac{N}{2^{u}}}^{(s)}(t) S_{n+\frac{1}{2} \chi(u+1)}^{(0)}(t) \\
& \quad=2^{-\frac{u}{2}} \sum_{l_{1}, l_{2}, \ldots, l_{u}=0}^{1}(-1)^{l_{1}} C_{n+N}^{(0)} \sum_{k=1}^{u} l_{k} 2^{k-u-1}(t) .
\end{aligned}
$$

Next, consider $u=\log _{2} N$. By invoking (32) $\log _{2} N$ times and (34) $\log _{2} N$ times, respectively, we have from (7) that

$$
\begin{aligned}
& \prod_{k=1}^{\log _{2} N} P_{\frac{N}{2^{k}}}^{(c)}(t) C_{n+\frac{1}{2} \chi\left(\log _{2} N\right)}^{(1)}(t) \\
& =2^{-\frac{1}{2} \log _{2} N} \sum_{l_{1}, l_{2}, \ldots, l_{\log _{2} N}=0}^{1} C_{N \sum_{k=1}^{(0)} \log _{2} N} l_{k} 2^{k-\log _{2} N-1}(t) \\
& \begin{array}{l}
\prod_{k=1}^{\log _{2} N} P_{\frac{N}{2^{k}}}^{(c)}(t) S_{n+\frac{1}{2} \chi\left(\log _{2} N\right)}^{(1)}(t) \\
=2^{-\frac{1}{2} \log _{2} N} \sum_{l_{1}, l_{2}, \ldots, l_{\log _{2} N}=0}^{1} S_{N \sum_{k=1}^{\log _{2} N} l_{k} 2^{k-\log _{2} N-1}}^{(0)}(t) .
\end{array}
\end{aligned}
$$

If $\left(l_{\log _{2} N}, l_{\log _{2} N-1}, \ldots, l_{1}\right)$ is used to denote the binary representation of an integer $v$, (41)-(44) can be translated to yield (11) for $\Theta_{u}^{(c)}$ s. ${ }^{5}$

\section{Proof of Proposition 3}

With $\mathcal{W}_{L}$ encoding, (15) and (18) can be rewritten as

$$
B_{n, k}=\vartheta_{n} \sum_{l=1}^{L} 2^{-\frac{l}{2}}(-1)^{1+\psi_{l, n}} D_{\chi(l)+\left\lfloor n / 2^{l}\right\rfloor, k} .
$$

When $u=1$, we have from (45) that

$$
\begin{gathered}
\sum_{v=0}^{2^{u}-1} \vartheta_{n 2^{u}+v}^{*} G_{n 2^{u}+v, \chi(u)+n}^{(\mathcal{W})} B_{n 2^{u}+v, k}=\sum_{v=0}^{1}\left\{\frac{1}{2} D_{\left\lfloor\frac{2 n+v}{2}\right\rfloor, k}\right. \\
\left.+\sum_{l=2}^{L} 2^{-\frac{l+1}{2}}(-1)^{1+\psi_{l, 2 n+v}} \cdot D_{\chi(l)+\left\lfloor\frac{2 n+v}{2^{l}}\right\rfloor, k}\right\}
\end{gathered}
$$

for $n \in \mathcal{Z}_{N / 2}$. Using $\psi_{l, 2 n+v}=\psi_{l, 2 n}+v$ and $\left\lfloor(2 n+v) / 2^{l}\right\rfloor=$ $\left\lfloor n 2^{1-l}\right\rfloor$ for $v \in \mathcal{Z}_{2}$ in (46) proves (28) for $u=1$. Next, when $u \in\{2,3, \ldots, L\}$, we further have from (45) that

$$
\begin{gathered}
\sum_{v=0}^{2^{u}-1} \vartheta_{n 2^{u}+v}^{*} G_{n 2^{u}+v, \chi(u)+n}^{(\mathcal{W})} B_{n 2^{u}+v, k} \\
=2^{-\frac{u}{2}} \sum_{v=0}^{2^{u}-1}(-1)^{1+\psi_{u, v}} B_{n 2^{u}+v, k} \\
=2^{-\frac{u+1}{2}} \sum_{x=0}^{2^{u-1}-1} \sum_{y=0}^{1}(-1)^{1+\psi_{u, 2 x+y}}
\end{gathered}
$$

${ }^{5}$ For notational simplicity, we delete the minus sign in the basis signals given by (41) when representing $\Theta_{u}^{(c)}$ in (11) with $1 \leq u \leq \log _{2} N-1$. Because the negative of a basis signal is still a basis signal, this deletion does not virtually affect the nature of the basis set in (11). 


$$
\begin{aligned}
& \times D_{\left\lfloor\frac{n 2^{u}+2 x+y}{2}\right\rfloor, k}+\sum_{l=2}^{L} F_{l, u}^{(1)} \\
= & \sum_{l=2}^{L} F_{l, u}^{(1)}
\end{aligned}
$$

for $n \in \mathcal{Z}_{N / 2^{u}}$, in which we have defined

$$
F_{l, u}^{(1)} \triangleq 2^{-\frac{u+l}{2}} \sum_{v=0}^{2^{u}-1}(-1)^{\psi_{u, v}+\psi_{l, n 2^{u}+v}} D_{\chi(l)+\left\lfloor\left(n 2^{u}+v\right) / 2^{l}\right\rfloor, k} .
$$

In deriving (47), the second equality stems from the decomposition $v=2 x+y$ for $x \in \mathcal{Z}_{2^{u-1}}$ and $y \in \mathcal{Z}_{2}$, and the third equality results because $\psi_{u, 2 x+y}=\psi_{u, 2 x}+y$ and $\left\lfloor\left(n 2^{u}+\right.\right.$ $2 x+y) / 2\rfloor=n 2^{u-1}+x$ for $y \in \mathcal{Z}_{2}$. Because $v<n 2^{u}$, $\psi_{l, n 2^{u}+v}=\psi_{l, n 2^{u}}+\psi_{l, v}$ holds and (48) becomes

$$
\begin{aligned}
F_{l, u}^{(1)}=2^{-\frac{u+l}{2}}(-1)^{\psi_{l, n 2^{u}}} \sum_{v=0}^{2^{u}-1}( & -1)^{\psi_{u, v}+\psi_{l, v}} \\
& \times D_{\chi(l)+\left\lfloor\left(n 2^{u}+v\right) / 2^{l}\right\rfloor, k} .
\end{aligned}
$$

Consider (49) in three different cases. When $l>u,\left\lfloor\left(n 2^{u}+\right.\right.$ $\left.v) / 2^{l}\right\rfloor=\left\lfloor n 2^{u-l}\right\rfloor$ holds and (49) becomes

$$
F_{l, u}^{(1)}=2^{-\frac{u+l}{2}}(-1)^{\psi_{l, n 2^{u}}} D_{\chi(l)+\left\lfloor n 2^{u-l}\right\rfloor, k} \sum_{v=0}^{2^{u}-1}(-1)^{\psi_{u, v}+\psi_{l, v}} .
$$

Because $\psi_{u, v}+\psi_{l, v}$ is even when $v<2^{u-1}$ and odd otherwise, (50) results in $F_{l, u}^{(1)}=0$. When $l<u$, the decomposition $v=$ $2^{l} x+y$ for $x \in \mathcal{Z}_{2^{u-l}}$ and $y \in \mathcal{Z}_{2^{l}}$ can be used to establish $\left\lfloor\left(n 2^{u}+v\right) / 2^{l}\right\rfloor=n 2^{u-l}+x, \psi_{u, v}=\psi_{u, 2^{l} x}+\psi_{u, y}$ and $\psi_{l, v}=\psi_{l, y}$. Thus, (49) becomes

$$
\begin{aligned}
\left.F_{l, u}^{(1)}=2^{-\frac{u+l}{2}(-1)^{\psi_{l, n 2^{u}}} \sum_{x=0}^{2^{u-l}-1}(}-1\right)^{\psi_{u, x 2^{l}}} D_{\chi(l)+n 2^{u-l}+x, k} \\
\\
\times \sum_{y=0}^{2^{l}-1}(-1)^{\psi_{u, y}+\psi_{l, y}} .
\end{aligned}
$$

Since $\psi_{u, y}+\psi_{l, y}$ is even when $y<2^{l-1}$ and it is odd otherwise, (51) yields $F_{l, u}^{(1)}=0$. When $l=u$, the identities $\psi_{l, n 2^{u}}=0$, $\psi_{u, v}=\psi_{l, v}$, and $\left\lfloor\left(n 2^{u}+v\right) / 2^{l}\right\rfloor=n$ hold, and thus simplify (49) to $F_{u, u}^{(1)}=D_{\chi(u)+n, k}$. Using these results in (47) proves (28) for $u \in\{2,3, \ldots, L\}$.

\section{E. Proof of Proposition 4}

With $\mathcal{V}_{L}$ encoding, (15) and (18) can be rewritten as

$$
B_{n, k}=\vartheta_{n} \sum_{l=1}^{L} 2^{-\frac{l}{2}} \phi_{l,\left\lfloor\frac{n}{N 2-l}\right\rfloor} D_{\chi(l)+\langle n\rangle_{N 2-l}, k}
$$

where $\langle n\rangle_{K}$ denotes the modulo- $K$ value of $n$. Using (52), we have for $n \in \mathcal{Z}_{N / 2^{u}}$

$$
\sum_{v=0}^{2^{u}-1} \vartheta_{n+\frac{N}{2^{u}} v}^{*} G_{n+\frac{N}{2^{u}} v, \chi(u)+n}^{(\mathcal{V})} B_{n+\frac{N}{2^{u}} v, k}=2^{-\frac{u}{2}} \sum_{l=1}^{L} 2^{-\frac{l}{2}} F_{l}^{(2)}
$$

where we have defined

$$
F_{l}^{(2)} \triangleq \sum_{v=0}^{2^{u}-1} \phi_{u, v} \phi_{l,\left\lfloor\frac{n+v N 2^{-}-u}{N 2^{-l}}\right\rfloor} D_{\chi(l)+\left\langle n+v N 2^{-u}\right\rangle_{N 2^{-l}}, k} .
$$

$F_{l}^{(2)}$ can be further simplified in three different cases. When $l=u$, the identities $\left\lfloor\left(n+v N 2^{-u}\right) /\left(N 2^{-l}\right)\right\rfloor=v$ and $\langle n+$ $\left.v N 2^{-u}\right\rangle_{N 2^{-l}}=n$ hold, and thus yield $F_{u}^{(2)}=2^{u} D_{\chi(l)+n, k}$. When $l>u,\left\lfloor\left(n+v N 2^{-u}\right) /\left(N 2^{-l}\right)\right\rfloor=\left\lfloor n /\left(N 2^{-l}\right)\right\rfloor+v 2^{l-u}$ and $\left\langle n+v N 2^{-u}\right\rangle_{N 2^{-l}}=\langle n\rangle_{N 2^{-l}}$. In the case

$$
F_{l}^{(2)}=\sum_{v=0}^{2^{u}-1} \phi_{u, v} \phi_{l,\left\lfloor\frac{n}{N 2^{-l}}\right\rfloor+v 2^{l-u}} D_{\chi(l)+\langle n\rangle_{N 2-l}, k}
$$

sums to zero, since $\left.\phi_{l,\left\lfloor n /\left(N 2^{-l}\right)\right\rfloor+v 2^{l-u}}=\phi_{l,\left\lfloor n /\left(N 2^{-l}\right)\right.}\right\rfloor$ does not vary with $v$ for $l>u$ and $\sum_{v=0}^{2^{u}-1} \phi_{u, v}=\sum_{v=0}^{2^{u}-1}(-1)^{\varsigma_{v}}=$ 0 for $u<\log _{2} N$. When $l<u$, the decomposition $v=2^{u-l} y+$ $x$ for $x \in \mathcal{Z}_{2^{u-l}}$ and $y \in \mathcal{Z}_{2^{l}}$ can be used to establish the identities $\phi_{u, v}=\phi_{u, x},\left\lfloor\left(n+v N 2^{-u}\right) /\left(N 2^{-l}\right)\right\rfloor=\left\lfloor\left(n /\left(N 2^{-l}\right)\right)+\right.$ $\left.x 2^{l-u}\right\rfloor+y$, and $\left\langle n+v N 2^{-u}\right\rangle_{N 2^{-l}}=\left\langle n+x N 2^{-u}\right\rangle_{N 2^{-l}}$. In the case

$$
\begin{aligned}
F_{l}^{(2)}=\sum_{x=0}^{2^{u-l}-1} \phi_{u, x} D_{\chi(l)+\langle n+} & \left.x N 2^{-u}\right\rangle_{N 2-l}, k \\
& \times \sum_{y=0}^{2^{l}-1} \phi_{l,\left\lfloor\frac{n}{N 2-l}+x 2^{l-u}\right\rfloor+y}
\end{aligned}
$$

sums to zero since the inner sum is zero for $l<\log _{2} N$. Using the results in (53) proves (29).

\section{REFERENCES}

[1] R. W. Chang, "Synthesis of band-limited orthogonal signals for multichannel data transmission," Bell Syst. Tech. J., vol. 45, pp. 1775-1796, Dec. 1966.

[2] B. R. Saltzberg, "Performance of an efficient parallel data transmission system," IEEE Trans. Commun. Technol., vol. COM-15, no. 12, pp. 805-811, Dec. 1967.

[3] B. Hirosaki, "An orthogonally multiplexed QAM system using the discrete Fourier transform," IEEE Trans. Commun., vol. COM-29, no. 7, pp. 982-989, Jul. 1981.

[4] J. A. C. Bingham, "Multicarrier modulation for data transmission: an idea whose time has come," IEEE Commun. Mag., vol. 28, pp. 5-14, May 1990.

[5] K. Zhong, T. Tjhung, and F. Adachi, "A general SER formula for an OFDM system with MDPSK in frequency domain over Rayleigh fading channels," IEEE Trans. Commun., vol. 52, no. 4, pp. 584-594, Apr. 2004.

[6] X. G. Xia, "Precoded and vector OFDM robust to channel spectral nulls and with reduced cyclic prefix length in single transmit antenna systems," IEEE Trans. Commun., vol. 49, no. 8, pp. 1363-1374, Aug. 2001

[7] Z. Wang, S. Zhou, and G. B. Giannakis, "Joint coding-precoding with low-complexity turbo-decoding," IEEE Trans. Wireless Commun., vol. 3 , no. 3, pp. 832-842, May 2004.

[8] M. Debbah, P. Loubaton, and M. de Courville, "Asymptotic performance of successive interference cancellation in the context of linear precoded OFDM systems," IEEE Trans. Commun., vol. 52, no. 9, pp. 1444-1448, Sep. 2004.

[9] B. Muquet, Z. Wang, G. B. Giannakis, M. de Courville, and P. Duhamel, "Cyclic prefixing or zero padding for wireless multicarrier transmissions?," IEEE Trans. Commun., vol. 50, no. 12, pp. 2136-2148, Dec. 2002.

[10] Y. Zhao, J. D. Leclercq, and S. G. Häggman, "Intercarrier interference compression in OFDM communication systems by using correlative coding," IEEE Commun. Lett., vol. 2, no. 8, pp. 214-216, Aug. 1998. 
[11] A. N. Husna, S. Y. S. Kamilah, B. Ameruddin, and E. Mazlina, "Intercarrier interference (ICI) analysis using correlative coding OFDM system," in Proc. RF Microw. Conf., Subang, Selangor, Malaysia, Oct. 2004, pp. 235-237.

[12] K. Sathananthanm and C. Tellambura, "Coding to reduce both PAR and PICR of an OFDM signal," IEEE Commun. Lett., vol. 6, no. 8, pp. 316-318, Aug. 2002.

[13] S. K. Yusof and N. Fisal, "Correlative coding with clipping and filtering technique in OFDM systems," in Proc. ICICS-PCM Conf., Singapore, Dec. 2003, pp. 1456-1459.

[14] M. Sharif and B. Hassibi, "Existence of codes with constant PMEPR and related design," IEEE Trans. Signal Process., vol. 52, no. 10, pp. 2836-2846, Oct. 2004.

[15] C. D. Chung, "Correlatively coded OFDM," IEEE Trans. Wireless Commun., vol. 5, no. 8, pp. 2044-2049, Aug. 2006.

[16] C. D. Murphy, "Low-complexity FFT structures for OFDM transceivers," IEEE Trans. Commun., vol. 50, no. 12, pp. 1878-1881, Dec. 2002.

[17] R. Li and G. Stette, "Time-limited orthogonal multicarrier modulation schemes," IEEE Trans. Commun., vol. 43, no. 2-4, pp. 1269-1272, Feb.-Apr. 1995.

[18] L. Wei and C. Schlegel, "Synchronization requirements for multi-user OFDM on satellite mobile and two-path Rayleigh fading channels," IEEE Trans. Commun., vol. 43, no. 2-4, pp. 887-895, Feb.-Apr. 1995.

[19] A. Vahlin and N. Holte, "Optimal finite duration pulses for OFDM," IEEE Trans. Commun., vol. 44, no. 1, pp. 10-14, Jan. 1996.

[20] M. Faulkner, "The effect of filtering on the performance of OFDM systems," IEEE Trans. Veh. Technol., vol. 49, no. 5, pp. 1877-1884, Sep. 2000.

[21] C. D. Chung, "Orthogonally-multiplexed orthogonal amplitude modulation family," IEEE Trans. Commun., vol. 50, no. 3, pp. 415-428, Mar. 2002.

[22] - "Coherent and differentially coherent detections of orthogonally multiplexed orthogonal phase-modulated signals," IEEE Trans. Commun., vol. 51, no. 3, pp. 428-440, Mar. 2003.

[23] M. K. Simon, S. M. Hinedi, and W. C. Lindsey, Digital Communication Techniques. Englewood Cliffs, NJ: Prentice-Hall, 1994.

[24] S. M. Fleisher and S. Qu, "Quadrature frequency/phase modulation," IEEE Trans. Commun., vol. COM-43, no. 2-4, pp. 1513-1524, Feb. -Apr. 1995.

[25] K. Feher, Ed., Advanced Digital Communications: Systems and Signal Processing Techniques. Englewood Cliffs, NJ: Prentice-Hall, 1987.

[26] W. R. Bennett, Introduction to Signal Transmission. New York: McGraw-Hill, 1970.

[27] Network and Consumer Installation Interfaces-Asymmetric Digital Subscriber Line (ADSL) Metallic Interface, T1.413, ANSI, 1995.

[28] Digital Video Broadcasting (DVB-T); Framing Structure, Channel Coding and Modulation for Digital Terrestrial Television, ETS 300 744, ETSI, Dec. 2001.
[29] Wireless LAN Medium Access Control (MAC) and Physical Layer (PHY) Specifications: Higher Speed Physical Layer (PHY) Extension in the $5 \mathrm{GHz}$ Band, IEEE 802.11a/D5.0, IEEE, Apr. 1999.

[30] S. Coleri, M. Ergen, A. Puri, and A. Bahai, "Channel estimation techniques based on pilot arrangement in OFDM systems," IEEE Trans. Broadcast., vol. 48, no. 3, pp. 223-229, Sep. 2002.

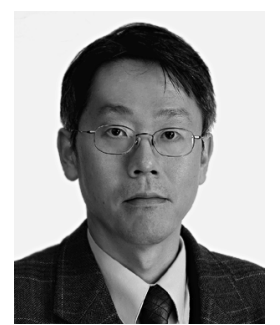

Char-Dir Chung (S'87-M'89-SM'03) received the B.S. degree in electrical engineering from the $\mathrm{Na}-$ tional Taiwan University, Taipei, Taiwan, R.O.C., in 1983, and the M.S. and Ph.D. degrees in electrical engineering from the University of Southern California, Los Angeles, in 1986 and 1989, respectively.

From 1989 to 1992, he was with the LinCom Corporation, Los Angeles, CA, where he worked on analytical and simulation modeling of scientific and military satellite communication systems. From 1992 to 2005, he was with the National Central University, Chung-Li, Taiwan, R.O.C., first as an Associate Professor, then as a Professor since 1996. At the National Central University, he founded the Advanced Communications Laboratory in 1998, the Graduate Institute of Communications Engineering in 2000, and the Department of Communications Engineering in 2003, and was the founding heads of these organizations. Since 2005, he has been on the faculty at the National Taiwan University, Taipei, Taiwan, R.O.C., where he is now a Professor of the Graduate Institute of Communications Engineering and the Department of Electrical Engineering. His current research interests are in digital modulation theory, wireless communications, and spread-spectrum communications. He has published more than 40 journal and conference papers and holds two patents in these areas. From 2000 to 2004, he was an Editor for the Journal of the Chinese Institute of Electrical Engineering, and since 2003, he has been an Editor for the Electrical Engineering Magazine of the same organization. In 2004, he was appointed the Chairman of the Wireless System Group of the National Science and Technology Program for Telecommunications, R.O.C., and the Chairman of the Taiwan Broadband Wireless Communications Industry Alliance, R.O.C. In the past decade, he has also actively served as a technical referee of numerous industrial product and technology development projects for several ROC government agencies.

Dr. Chung received the Group Achievement Award from the National Aeronautics and Space Administration, USA, in 1991, the Young Scientists Award from the International Union of Radio Science in 1993, the annual Research Award from the National Science Council, R.O.C., in 1992 and from 1994 to 2001, the Kentucky Colonel grade from the Commonwealth of Kentucky, USA, in 2003, and the FORMOSAT-2 Satellite Project Award from the National Space Center, R.O.C., in 2005. He served as the Chairman of the IEEE Information Theory Society, Taipei Chapter, from 1997 to 1999. 